\title{
Flooding edge or node weighted graphs
}

\author{
Fernand Meyer
}

Centre de Morphologie Mathématique

22 March 2013 


\section{Introduction}

Floodings are useful for:

- filtering images

- suppressing regional minima and filling them by lakes -> regularization and control of the watershed segmentation

Combined with the dual operator, the razings, they permit to construct powerful autodual filters (alternate sequential, flattenings and levelings) 


\section{Outline of the talk}

We flood two types of graphs:

- node weighted graphs with a ground level on the nodes

- edge weighted graph without ground level on the nodes

The flooding assigns a flooding to each node.

We characterize valid floodings on both types of graphs: lakes, regional minima lakes.

We show that flooding a node weighted graph is equivalent with flooding an edge weighted graph with appropriate edge weights 


\section{Outline of the talk}

We then introduce dominated floodings under a ceiling function. We present two classes of algorithms :

- shortest path algorithms for the ultrametric flooding distance

- direct construction of the flooding on the dendrogram of the closed balls of the flooding distance 


\section{Reminders on graphs}




\section{Graphs}

A non oriented graph $G=[N, E]: N=$ nodes ; $E=$,edges ; an edge $u \in E=$ a pair of vertices

A chain of length $n$ is a sequence of $n$ edges $L=\left\{e_{1}, e_{2}, \ldots, e_{n}\right\}$, with successive edges having a common node.

A path between two nodes $x$ and $y$ is a sequence of nodes $\left(n_{1}=x, n_{2}, \ldots, n_{k}=y\right)$ with successive nodes linked by an edge.

A cocycle is the set of all edges with one extremity in a subset $Y$ and the other in the complementary set $\bar{Y}$.

The subgraph spanning a set $A \subset N$ is the graph $G_{A}=\left[A, E_{A}\right]$, where $E_{A}$ are the edges linking two nodes of $A$.

The partial graph associated to the edges $E^{\prime} \subset E$ is $G^{\prime}=\left[N, E^{\prime}\right]$. 


\section{Weighted graphs}

In a graph $G=[N, E]$, edges and nodes may be weighted :

- $e_{i j}$ is the weight of the edge $(i, j)$

- $n_{i}$ the weight of the node $i$. The weights take their value in a completely ordered lattice $\mathcal{T}$. 


\section{Flat zones and regional minima on node weighted graphs}

A subgraph $G^{\prime}$ of a node weighted graph $G$ is a flat zone, if any two nodes of $G^{\prime}$ are connected by a path where all nodes have the same altitude. A subgraph $G^{\prime}$ of a graph $G$ is a regional minimum if $G^{\prime}$ is a flat zone and all neighboring nodes have a higher altitude 
Distances on a graph
Case of edge weighed graphs

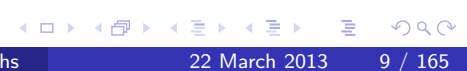




\section{Constructing distances on an edge weighted graph.}

Distances on an edge weighted graph have chains as support :

1) Definition of the weight of a chain, as a measure derived from the edge weights of the chain elements (example: sum, maximum, etc.)

2) Comparison of two chains by their weight. The chain with the smallest weight is called the shortest.

The distance $d(x, y)$ between two nodes $x$ and $y$ of a graph is $\infty$ if there is no chain linking these two nodes and equal to the weight of the shortest chain if such a chain exists.

Given three nodes $(x, y, z)$ the concatenation of the shortest chain $\pi_{x y}$ between $x$ and $y$ and the shortest chain $\pi_{y z}$ between $y$ and $z$ is a chain $\pi_{x z}$ between $x$ and $z$, whose weight is smaller or equal to the weight of the shortest chain between $x$ and $z$. To each distance corresponds a particular triangular inequality : $d(x, z) \leq$ weight $\left(\pi_{x y} \triangleright \pi_{y z}\right)$ where $\pi_{x y} \triangleright \pi_{y z}$ represents the concatenation of both chains. 


\section{Distance on an edge weighted graph based a the length of}

the shortest chain

Length of a chain: The length of a chain between two nodes $x$ and $y$ is defined as the sum of the weights of its edges.

Distance: The distance $d(x, y)$ between two nodes $x$ and $y$ is the minimal length of all chains between $x$ and $y$. If there is no chain between them, the distance is equal to $\infty$.

Triangular inequality : For $(x, y, z): d(x, z) \leq d(x, y)+d(y, z)$ 


\section{Distance on a graph based on the maximal edge weight}

along the chain

The weights are assigned to the edges, and represent their altitudes. Altitude of a chain: The altitude of a chain is equal to the highest weight of the edges along the chain.

Flooding distance between two nodes: The flooding distance fldist $(x, y)$ between nodes $x$ and $y$ is equal to the minimal altitude of all chains between $x$ and $y$. During a flooding process, in which a source is placed at location $x$, the flood would proceed along this chain of minimal highest altitude to reach the pixel $y$. If there is no chain between them, the level distance is equal to $\infty$.

Triangular inequality : For $(x, y, z): d(x, z) \leq d(x, y) \vee d(y, z)$ : ultrametric inequality 


\section{The flooding distance is an ultrametric distance}

An ultrametric distance verifies

$*$ reflexivity: $d(x, x)=0$

* symmetry: $d(x, y)=d(y, x)$

* ultrametric inequality: for all $x, y, z: d(x, y) \leq \max \{d(x, z), d(z, y)\}$ : the lowest lake containing both $x$ and $y$ is lower or equal than the lowest lake containing $x, y$ and $z$. 


\section{Distances on a graph : sum and maximum of the edge}

weights

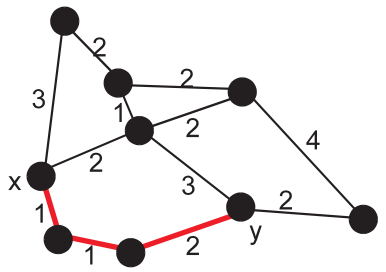

The shortest chain (sum of weights of the edges) between $x$ and $y$ is a red line and has a length of 4 .

The lowest chain (maximal weight of the edges) between $x$ and $y$ is a red line and a maximal weight of 2. A flooding between $x$ and $y$ would follow this chain. 
Flooding a topographic surface or flooding a graph 


\section{The region adjacency graph}

We will work with "neighborhood graphs" where the nodes are the catchment basins and the edges connect neighboring bassins. The edges are weighted by a dissimilarity measure between adjacent catchment basins; the simplest being the altitude of the pass-point between two basins.
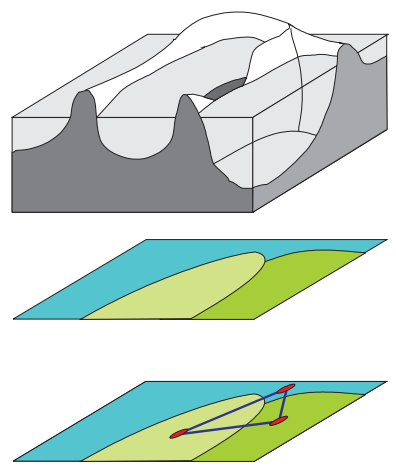

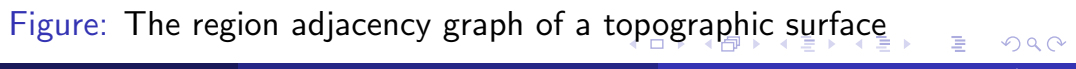
Fernand Meyer (Centre de Morphologie Math Flooding edge or node weighted graphs 


\section{Representation of a flooded topographic surface as a node weighted graph.}

An image may be considered as a topographic surface. The altitude of each pixel corresponds to its gray level. An image may be modelled by a graph, the nodes being the pixels and the edges connecting neighboring pixels. A first weight distribution $f$ represents the ground level.

For a flooded surface, the nodes hold a second weight $\tau \geq f$ equal to the flooding level.

The edges are not weighted. 


\section{Representation of a flooded RAG as an edge weighted}

graph

A physical interpretation of a flooded RAG: the nodes are tanks with infinite height and depth, their weight represent the height of the flooding in the tank. If two nodes are connected by a weighted edge, the corresponding tanks are linked by a pipe at an altitude of the weight. The pipes allow the water to pass from tank to tank, according the laws of hydrostatics. We call such a graph tank network (TN). The level in each tank is indicated in blue.

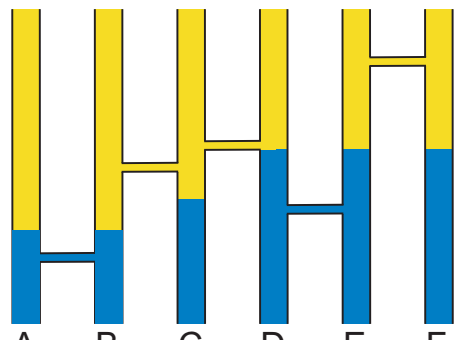

A $\quad$ B $\quad C \quad D \quad E$

$F$ 


\section{Flooding a topographic surface of its region adjacency}

graph
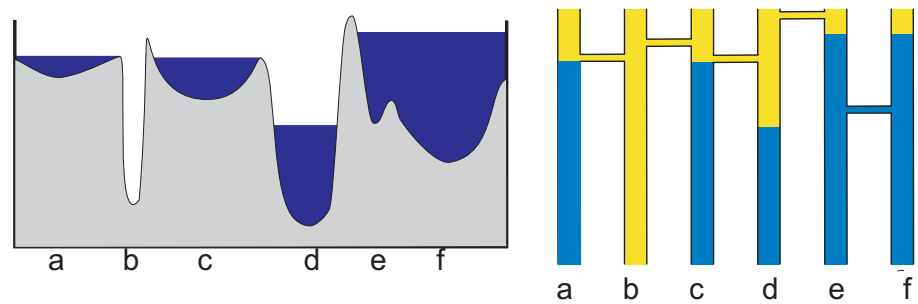

Figure: Flooding a topographic surface or flooding its region adjacency graph.

The flooding of a topographic surface is perfectly defined if one knows the flooding level in or above each catchment basin. The same flooding may be represented on the region adjacency graph by assigning to each node the flooding level in the corresponding basin. 


\section{Modelling the laws of hydrostatics in node and edge weighted tanks}

As flooding a topographic surface and flooding its RAG represent the same phenomenon, we have to find two models, one for node weighted graphs, the other for edge weighted graphs, expressing this same phenomenon 
Flooding a topographic surface or nodes weighted graph 


\section{Definition of a flooding of a function}

\section{Definition}

A function $g$ is a flooding of a function $f$ if and only if $g \geq f$ and for any couple of neighboring pixels $(p, q): g_{p}>g_{q} \Rightarrow g_{p}=f_{p}$

Fig. 4A presents a physically possible flooding. On the contrary the flooding in fig. $4 \mathrm{~B}$ is impossible, as the lake containing the pixel $p$ where $g_{p}>f_{p}$ is not limited by solid ground since $g_{p}>g_{q}$.
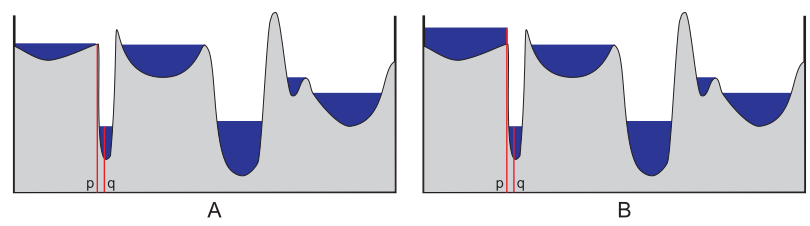

Figure: A possible and an impossible flooding 


\section{Flooding a node weighted graph}

Images are particular node weighted graphs, the pixels being the nodes. Neighboring pixels are linked by an unweighted edge. We now consider arbitrary node weighted graphs. The node weights $f_{i}$ indicate the ground level. The edges are not weighted. Such a topographic graph is flooded if the nodes are assigned a second family of weights indicating the level of the flooding at each node. 


\section{Flooding a node weighted graph}

A distribution $\tau$ of node weights will represent an effective flooding if it verifies a number of conditions of equilibrium:

- A flooding being always above the ground level: $\tau_{i} \geq f_{i}$.

- As there is nothing to prevent the water to flow from a higher to a lower position, an inequal level of water at two neighboring nodes $p$ and $q$ is impossible, except when the highest node is dry ; hence $\tau_{p}>\tau_{q} \Rightarrow \tau_{p}=f_{p}$ indicating that the highest level is dry, without water.

- Consequence 1: in a lake, the level of all nodes is the same.

- Consequence 2: floodings are connected operators : $f_{p}=f_{q} \Rightarrow \tau_{p}=\tau_{q}$ 


\section{Flooding an edge weighted graph}




\section{Flooding an edge weighted graph}

$G=[E, N]$ : a node and edge weighted graph, $E=$ edges, $N=$ nodes. The edges are weighted: the weight $e_{i j}$ of the edge $(i, j)$ represents the altitude at which a flood coming from one extremity may reach the other extremity of the edge.

The nodes also are weighted; $\tau_{i}$ represents the altitude of the flood at node $i$. 


\section{Flooding an edge weighted graph}

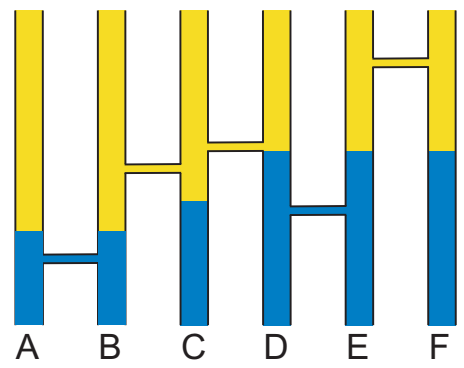

Figure:

We consider the nodes as vertical tanks of infinite height and depth : there is no ground level.

The weight $\tau_{i}$ represents the level of water in the tank $i$.

Two neighboring tanks $i$ and $j$ are linked by a pipe at an altitude $e_{i j}$ equal to the weight of the edge.

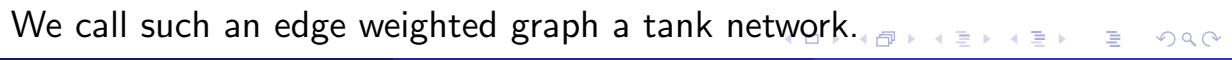




\section{Flooding an edge weighted graph}

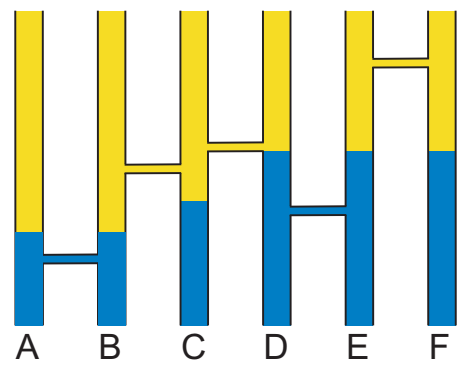

Laws of hydrostatics:

- if the level $\tau_{i}$ in the tank $i$ is higher than the pipe $e_{i j}$, then the levels is the same in both tanks $i$ and $j: \tau_{i}=\tau_{j}$.

- the level $\tau_{i}$ in the tank $i$ cannot be higher than the level $\tau_{j}$, unless $e_{i j} \geq \tau_{i}$. 


\section{Flooding an edge weighted graph}

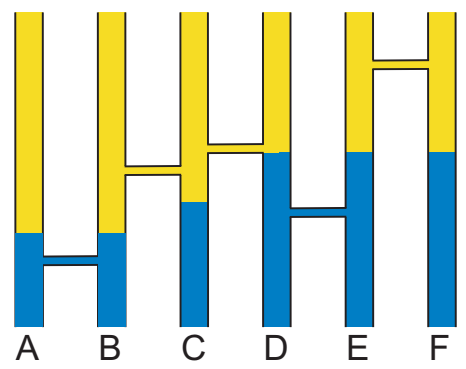

Figure: Tank and pipe network:

- $A$ and $B$ form a regional minimum with $\tau_{A}=\tau_{B}=\lambda ; e_{A B} \leq \lambda ; e_{B C}>\lambda$

- $B$ and $C$ have unequal levels but are separated by a higher pipe.

- $D$ and $E$ form a full lake, reaching the level of its lowest exhaust pipe $e_{C D}$

- E and $F$ have the same level ; however they do not form a lake, as they are linked by a pipe which is higher 


\section{Flooding an edge weighted graph}

\section{Definition}

The distribution $\tau$ of water in the pipes of the graph $[E, N]$ is a flooding of this graph, i.e. is a stable distribution of fluid if it verifies the criterion: for any couple of neighboring nodes $(p, q)$ we have:
$\left(\tau_{p}>\tau_{q} \Rightarrow e_{p q} \geq \tau_{p}\right)$
(criterion 1)

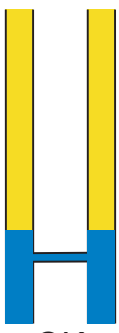

OK

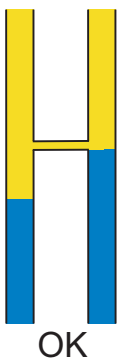

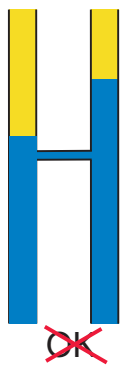

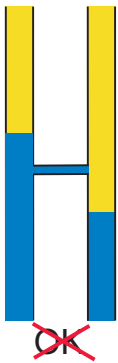

Figure: The water distribution marked OK are compatible with the laws of physics; the others are not. 


\section{Criteria}

$\left(\tau_{p}>\tau_{q} \Rightarrow e_{p q} \geq \tau_{p}\right) \Leftrightarrow\left(\operatorname{not}\left(\tau_{p}>\tau_{q}\right)\right.$ or $\left.e_{p q} \geq \tau_{p}\right) \Leftrightarrow\left(\tau_{p} \leq \tau_{q}\right.$ or $\left.\tau_{p} \leq e_{p q}\right) \Leftrightarrow$

$\left(\tau_{p} \leq \tau_{q} \vee e_{p q}\right) \quad$ (criterion 2)

$\Leftrightarrow\left(\tau_{p} \leq \tau_{q} \vee\right.$

$(p, q)$ neighbors

$\left(\tau_{q} \vee e_{p q}\right) \quad($ criterion 3$)$

\section{Remark}

The criterion $\left(\tau_{p}>\tau_{q} \Rightarrow e_{p q} \geq \tau_{p}\right)$ is equivalent with

$\left(e_{p q}<\tau_{p} \Rightarrow \tau_{p} \leq \tau_{q}\right)$. Hence if $e_{p q}<\tau_{p}$, we have $\tau_{p} \leq \tau_{q}$; so we also have $e_{p q}<\tau_{q}$ implying $\tau_{q} \leq \tau_{p}$; finally $\tau_{p}=\tau_{q}$.

\section{$\square$}


Flooding a topographic graph is the same as flooding an associated edge weighted graph 


\section{Flooding a TG is the same as flooding a TN}

$G_{n}=[E, N]$ : a topographic graph. Ground level $=f$. The edges are not weighted.

The lowest level of flood covering two neighboring nodes $p$ and $q$ is equal to $f_{p} \vee f_{q}$.

Consider now a second graph $G_{e}$ with the same structure but with edge weights $e_{p q}=f_{p} \vee f_{q}$.

Any flooding $\tau \geq f$ of $G_{e}$ verifies: for $(p, q)$ neighbors

$\left(\tau_{p} \leq \tau_{q} \vee e_{p q}\right) \Leftrightarrow\left(\tau_{p} \leq \tau_{q} \vee f_{q} \vee f_{p}\right) \Leftrightarrow\left(\tau_{p} \leq \tau_{q} \vee f_{p}\right)$ as $\tau_{q} \geq f_{q}$ But this last criterion characterizes a flooding of $G_{n}$.

\section{Theorem}

There is an equivalence between the floodings $\tau \geq f$ of $G_{e}$ of $G_{n}$ and the floodings $\tau \geq f$ of $G_{e}$, with edge weights $e_{p q}=f_{p} \vee f_{q}$. 


\section{Lakes of edge weighted graphs}




\section{Lakes of edge weighted graphs}

If a node $p$ has no neighboring node $q$ such that $\tau_{p}=\tau_{q}$, then $p$ is an isolated node and isolated lake.

Consider now two neighboring nodes $p$ and $q$ verifying $\tau_{p}=\tau_{q}$. Adding a drop of water at the node $p$ has no impact on node $q$, if there exists no path linking $p$ and $q$ with edge weights $\leq \tau_{p}, \tau_{q}$.

We define a binary relation between neighboring pixels

$p, q: p \sim q \Leftrightarrow \tau_{p}=\tau_{q}$ and $e_{p q} \leq \tau_{p}, \tau_{q}$.

\section{Lemma}

If we cut all edges which do not verify $p \sim q$, we get a partial graph $\widetilde{G}$; the connected components of $\widetilde{G}$ are the lakes of the graph $G$. 


\section{Lakes of node weighted graphs}




\section{Lakes of node weighted graphs}

Consider now a topographic graph $G_{n}$ with a ground level $f$ and its derived tank network $G_{e}$ with edge weights $e_{p q}=f_{p} \vee f_{q}$. Any flooding of $G_{n}$ also is a flooding of $G_{e}$. Applying the definition of lakes given above we distinguish two cases:

- $p$ is an isolated node : it has no neighboring node $q$ such that $\tau_{p}=\tau_{q}$

- $p$ is not isolated, and has at least one neighbor $q$ such that $\tau_{p}=\tau_{q}$. As $\tau \geq f$, we have $\tau_{p}=\tau_{q}=\tau_{p} \vee \tau_{q} \geq f_{p} \vee f_{q}=e_{p q}$. This shows that $\tau_{p}=\tau_{q} \Rightarrow p \sim q$. This shows that the lakes of $G_{n}$ simply are its flat zones.

\section{Definition}

The lakes of a TG are its flat zones, that is maximal connected components of nodes with the same altitude. 


\section{Lakes of node weighted graphs}

A lake on a topographic graph is dry if it has a uniform altitude at the ground level. It is a wet lake, if it contains at least one pixel $p$ for which $\tau_{p}>f_{p}$. The two following lemmas concern wet lakes. The first is a reinterpretation of a lemma established for TN.

\section{Lemma}

If two neighboring nodes $p$ and $q$ verify $\tau_{p}>e_{p q}=f_{p} \vee f_{q}$, then $\tau_{p}=\tau_{q}$.

The second derives from criterion TG-1.

\section{Lemma}

If two neighboring nodes $p$ and $q$ verify $\tau_{p}>f_{p}$ and $\tau_{q}>f_{q}$, then $\tau_{p}=\tau_{q}$.

Proof: $\left\{\tau_{p}>\tau_{q} \Rightarrow \tau_{p} \leq f_{p}\right\} \Leftrightarrow\left\{\tau_{p}>f_{p} \Rightarrow \tau_{p} \leq \tau_{q}\right\}$. Applying the last implication to $\tau_{p}>f_{p}$ and $\tau_{q}>f_{q}$ yields $\tau_{p} \leq \tau_{q}$ and $\tau_{p} \geq \tau_{q}$, which together gives $\tau_{p}=\tau_{q}$. 


\section{Regional minima lakes and full lakes}




\section{Full lakes and regional minimum lakes in a tank network}

What happens at the boundary of a lake $X$ in a tank network? Consider 2 neighboring pixels $(p, q), p$ being inside a lake of altitude $\lambda$ and $q$ outside. These pixels do not verify $p \sim q: e_{p q}>\tau_{p}$ and $\tau_{p} \neq \tau_{q}$ :

- if $e_{p q}>\tau_{p}$ and one has to climb for going from $p$ to $q$.

- else $e_{p q} \leq \tau_{p}$ implying $\tau_{q} \leq \tau_{p} \vee e_{p q}=\tau_{p}$. As $\tau_{p} \neq \tau_{q}$ we have $\tau_{q}<\tau_{p}$, which implies $\tau_{p} \leq e_{p q}$. Thus $\tau_{p}=e_{p q}$ and $\tau_{q}<\tau_{p}$, indicating the $q$ is an exhaust node of the lake, and the lake $X$ is a full lake.

In other terms, in a lake without exhaust edges, all outgoing edges are higher than the level of the lake. Such a lake is called regional minimum lake. A lake with one or several exhautst edges is called full lake. Adding a drop of water to a full lake provokes an overflow through the exhaust edges.

\section{Definition}

A regional minimum of a tank network is a lake with all outgoing edges, or cocycle edges having a higher altitude. 


\section{Full lakes and regional minimum lakes in a tank network}

\section{Definition}

A lake of level $\lambda$ of the flooding of a tank network is a full lake, if there exists an an exhaust edge from an inside node $p$ to an outside node $q$ verifying $\tau_{p}=e_{p q}=\lambda>\tau_{q}$.

\section{Lemma}

Each regional minimum of the flooding of a tank network contains a regional minimum of the tank network itself or is an isolated regional minimum. .

Proof: Either $X=\{p\}$ is an isolated regional minimum node $p$ with all adjacent edges having a weight $>\lambda$. If $X$ contains inside edges, and $(p, q)$ is the edge for which $e_{p q}$ is minimal, then the maximal connected component containing $p$ with edge weights equal to $e_{p q}$ is a regional minimum of the graph. 


\section{Full lakes and regional minimum lakes in topographic graph}

A couple of neighboring nodes belongs to the cocycle of a lake $X, p \in X$ and $q \notin X$ only if $\tau_{p} \neq \tau_{q}$. Either $\tau_{p}>\tau_{q}$. Or $\tau_{p}>\tau_{q}$ implying $\tau_{p}=f_{p}$ and the lake is a full lake having an exhaust node $p$. In the graph $G_{e}$ we have $e_{p q}=f_{p} \vee f_{q}=\tau_{p}$ as $f_{p}=\tau_{p}>\tau_{q} \geq f_{q}$.

We get the two following definitions.

\section{Definition}

A regional minimum is a lake for which the ground level of all outside neighbors has a higher altitude.

\section{Definition}

A lake of the flooding of a topographic surface is a full lake of altitude $\lambda$ if there exist two neighboring nodes $p$ inside the lake and $q$ outside, such that $\tau_{p}<\tau_{q}=f_{q}$.

Each regional minimum of the flooding of a topographic graph contains a

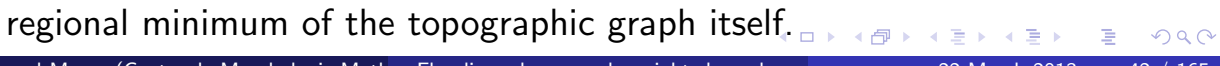




\section{Among all possible flooidngs, chosing one}




\section{Specifying a particular flooding among all possible ones}

Many floodings of a topographic surface of of an edge weighted graph are possible. In order to specify a particular flooding we have to add other criteria. For instance the lowest flooding for which each lake is a full lake or has a surface area higher than a given threshold specifies the so-called area flooding. we are interested by the highest flooding under a ceiling function $\omega$.
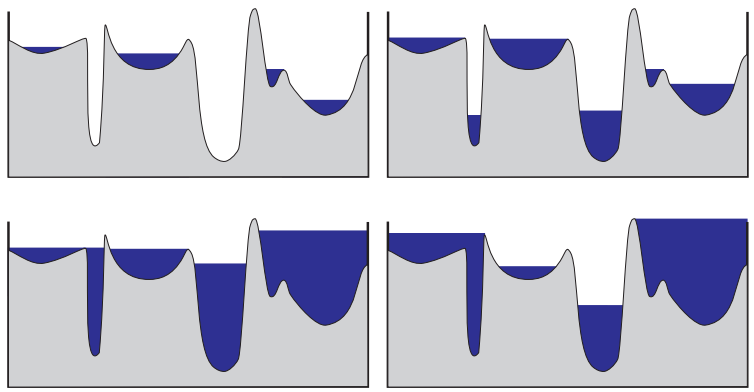

Figure: Various flood distribution on the same topographic surface 


\section{The algebra of floodings}

\section{Lemma}

If $\tau$ and $v$ are two floodings of a node or edge weighted graph $G$, then $\tau \vee v$ and $\tau \wedge v$ also are floodings of $G$.

Hence the family of floodings of the graph $G_{e}$ or $G_{n}$ below the function $\omega$ is closed by supremum. This supremum is itself a flooding and is below $\omega$. For this reason it is the highest flooding of the graph $G_{e}$ or $G_{n}$ below $\omega$. 


\section{Illustration}

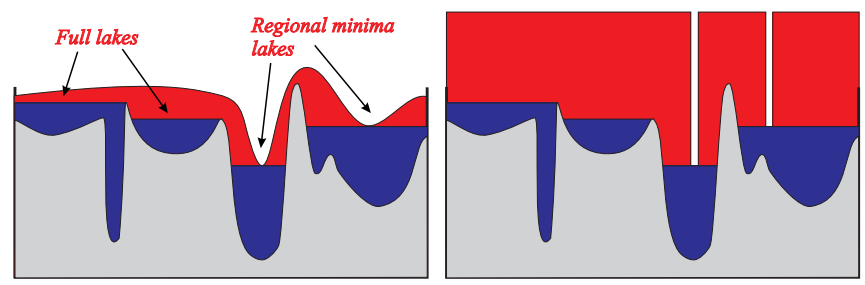

Figure: The highest flooding of a topographic surface below a ceiling functino (in red). The ceiling function on the left and on the right yield the same flooding, as they constrain the level regional minima lakes at identical levels ; all other lakes being full lakes.

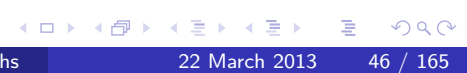




\section{Marker driven watershed segmentation}

The watershed of the gradient contains the contours of the image. The regions to segment contain each a marker. A ceiling function equal to the gradient image on the markers and equal to $\infty$ everywhere else is constructed. The highest flooding under this ceiling function has regional minima lakes at the position of the markers and full lakes everywhere else. The watershed of the flooded surface gives the result.
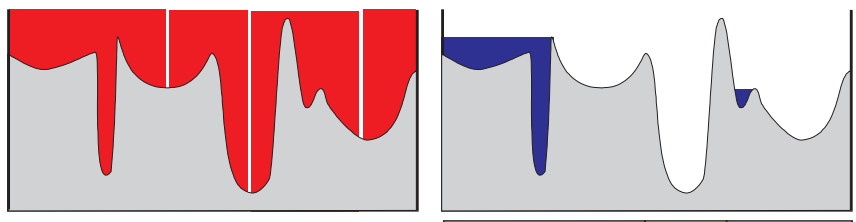

Figure: Left: a ceiling function with three minima above a topographic surface Right: the highest flooding of the topographic surface below the ceiling function. It contains three regional minima lakes. The watershed partition of this function is indicated below, each region labeled with a distinct color and contains one minimum of the ceiling function. 
The regional minimum lakes of dominated floodings 


\section{Regional minima of dominated edge weighted graphs}

Consider a flooding $\tau$ of a tank network and $X$, one of its lakes of altitude $\lambda$. Each node $p$ of $X$ verifies $\tau_{p}=\lambda$.

If there exists a pair of nodes, $s \in X$ and $t \notin X$, such that $\tau_{t}<\lambda$ and $e_{s t}=\lambda$ then $X$ is a full lake and the edge $(s, t)$ an exhaust edge for $X$. Such a lake cannot have a higher altitude than its exhaust edges, and the constraining function plays no role in the level of the lake.

On the contrary, if all edges of the cocycle of $X$ have an altitude above $\lambda$, then $X$ is a regional minimum of the flooding $\tau$. If the level $\lambda$ of the lake cannot become higher, it is because it is constrained by at least one node of the ceiling function $\omega$ at altitude $\lambda$. This node cannot be any node, as stated now.

\section{Theorem}

Any regional minimum lake of the highest flooding of a graph $G_{e}$ with edge weights e, below a ceiling function $\omega$, contains a regional minimum lake of the graph with edge weights $\delta_{e n} \omega \vee$ e and node weights $\omega$. 


\section{Regional minima of dominated node weighted graphs}

\section{Theorem}

Any regional minimum lake of the highest flooding of a graph $G$ with node weights $f$, below a ceiling function $\omega$, contains a regional minimum lake of the function $\omega$.

A regional minimum lake $X$ on a topographic graph has a uniform flooding level $\lambda$ and all its neighboring nodes have a flooding level $>\lambda$. The level of $X$ could be higher, were it not constrained by the ceiling function $\omega$. There exists a node $p \in X$ for which $\omega_{p}=\tau_{p}=\lambda$. The connected component $Y$ of nodes for which $\omega=\lambda$ contains $p$ and is included in $X$, as for each outside neighbor $q$ of $X$, we have $\omega_{q} \geq \tau_{q}>\lambda$. On $X$ we have $\omega \geq \tau=\lambda$, showing that $Y$ has no lower neighbor. Thus $Y$ is a regional minimum of the ceiling function. 


\section{Algorithmic consequences}

The highest flooding of $f$ under $\omega$ if made of lakes and of dry zones, where the flooding equals the ground level. The lakes themselves are divided between full lakes and regional minima lakes. The level of the full lakes is solely determined by the altitude of the lowest pass point surounding the lakes. The level of regional minima lakes is determined by the level of the regional minima of the ceiling function. In fact, the blocking effect is the same whatever the size of this regional minimum ; a single point is sufficient.

Replace the ceiling function $\omega$ by a function equal to $\omega$ on at least one node of each regional minimum produces the same dominated flooding. 


\section{Considering the flooding process itself}




\section{Observing the progression of a flooding process on an edge weighted graph.}

We place a source pouring water at a node $\Omega$ of an edge weighted graph $G_{e}$ and flood the graph. We are interested by the level of the flood at each other node of the graph when it reaches for the first time this node. If $p$ is a node of the graph, the flood coming from $\Omega$ will reach $p$ following the easiest path: among all paths between $\Omega$ and $p$, it follows the path for which the highest edge is the lowest. This value constitutes precisely the ultrametric distance $d(\Omega, p)$ between $\Omega$ and $p$. 


\section{The result of a flooding process is a flooding}

Consider the shortest path between $\Omega$ and $p$. The value $\tau_{p}$ is the weight of the highest edge between $\Omega$ and $p$. The last node on this shortest path is a node $q$. If the highest edge on the path is $(q, p)$, then $\tau_{p}=e_{p q}$. If not $\tau_{p}=\tau_{q} \geq e_{p q}$ as the highest edge between $\Omega$ and $p$ of the path $\pi$ lies between $\Omega$ and $q$. In all cases we have $\tau_{p}=\tau_{q} \vee e_{p q}$. Consider now any other neighboring node $s$ of $p$. The path obtained by concatenating the shortest path between $\Omega$ and $s$ and the edge $(s, p)$ is not necessarily the shortest path between $\Omega$ and $p$, hence $\tau_{p} \leq \tau_{s} \vee e_{p s}$.

\section{Theorem}

The shortest ultrametric distance of each node $p$ of an edge weighted graph to a particular node $\Omega$ is a flooding of this graph. 


\section{The flooding process produces a dominated flooding}

Let $I$ be the subset of nodes for which $d(\Omega, i)=\omega_{i}$. If the geodesic path between $\Omega$ and a node $p$ passes through $i$, then $\tau_{p}=d(\Omega, p)=e_{\Omega \omega_{i}} \vee d(i, p)=\omega_{i} \vee d(i, p)$. For any node $q$ we have $\tau_{q}=\bigwedge_{i \in I} \omega_{i} \vee d(i, q)$.

This shows that $\tau$ is the highest possible flooding of $G_{e}$ on all nodes $\omega_{i}$ and also on all other nodes. Suppressing the node $\Omega$ and all edges linking $\Omega$ with another node of $G_{e}$ produces a graph $G^{\prime}$ for which $\tau$ is the highest flooding dominated by $\omega$. 


\section{Inversely, each dominated flooding is produced by a}

flooding.

Any dominated flooding verifies $\tau_{p} \leq \bigwedge_{q \text { neighbor of } p}\left(\tau_{q} \vee e_{p q}\right)$ and $\tau_{p} \leq \omega_{p}: \tau_{p} \leq \omega_{p} \wedge$ $q$ neighbor of $p$ $\left(\tau_{q} \vee e_{p q}\right)$.

The highest of them verifies $\tau_{p}=\omega_{p} \wedge$ $q$ neighbor of $p$

$\bigwedge \quad\left(\tau_{q} \vee e_{p q}\right)$

Adding to the graph $G$ a dummy node $\Omega$ with a weight $\tau_{\Omega}=0$ linked by a dummy edge $(\Omega, p)$ with a weight $\omega_{p}$ produces a graph $\widehat{G}$. Rewritten as $\tau_{p}=\left(\tau_{\Omega} \vee e_{\Omega p}\right) \wedge \wedge\left(\tau_{q} \vee e_{q p}\right)$, this formula is the expression of $q$ neighbor of $p$ the algorithm of Berge for computing the shortest ultrametric distance of each node to $\Omega$ in the augmented graph $\widehat{G}$.

The algorithm of Berge expresses that the shortest path between $\Omega$ and $p$ is $e_{\Omega p}=\omega_{p}$ if the path is simply the edge $(\Omega, p)$ or it is equal to $\left(\tau_{s} \vee e_{p s}\right)$ if the path passes through the neighbor $s$ of $p$, and if $\left(\tau_{q} \vee e_{q p}\right)$ takes its smallest value for $q=s$. 


\section{Inversely, each dominated flooding is produced by a}

flooding.
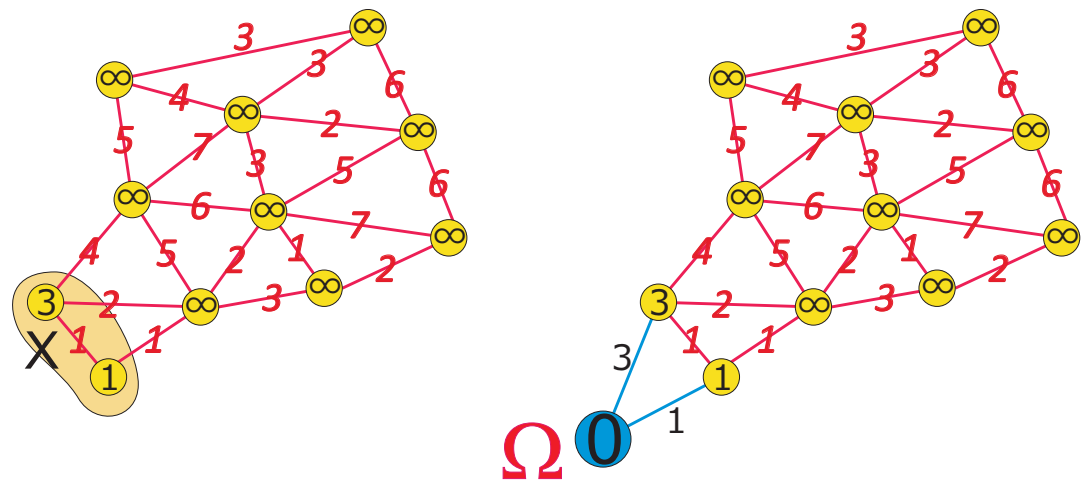

Figure: Adding a dummy node linked to each node $x$ in $X$ by an edge weighted by the offset at $x$.

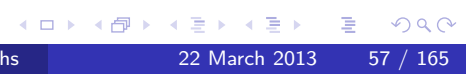




\section{Inversely, each dominated flooding is produced by a flooding.}

\section{Theorem}

The highest flooding of the graph $G$ below a function $\omega$ defined on the nodes is the shortest distance of each node to $\Omega$ in augmented graph $\widehat{G}$.

If the flooding is not constrained on a node $p$, i.e. $\omega_{p}=\infty$, then it is not necessary to link the node $p$ with the dummy node $\Omega$, as the highest flooding will reach $p$ through one of its neighboring nodes. 


\section{Pruning the graph and getting the same result}




\section{Edge weighted graphs}

Each dominated flooding results in a flood distribution verifying criterion DF-2: $\tau_{q}=\bigwedge_{i \in N} \omega_{i} \vee d(i, q)$.

The ultrametric distance $d(i, q)$ is the weight of the highest edge in a path $\pi$ of lowest sup-section between $i$ and $q$. Consider now each edge $(p, q)$ of the path $\pi$. If it belongs to $\pi$ we keep it. If not we may replace it in $\pi$ by the unique path between $p$ and $q$ contained in $T$, as all edges along this path have a weight $\leq e_{p q}$; these substitutions produce paths with the same sup-section. In other words, the edges of the tree $T$ are sufficient for computing the ultrametric distances of the graph 


\section{The flooding always follows the union of minimum spanning trees}

\section{Illustration :}

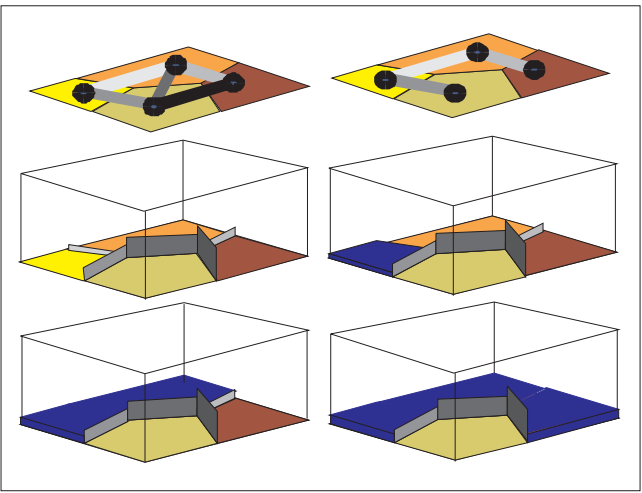




\section{Node weighted graphs}

Any flooding $\tau \geq f$ on a node weighted graph $G_{n}$ also is a flooding of the derived edge weighted graph $G_{e}$ with edge weights $\delta_{e n} e$. The preceding results apply. Any flooding $\tau \geq f$ of a MST of $G_{e}$ also is a flooding of $G_{e}$ and of $G_{n}$. 


\section{Algorithmic consequences}

This result has interesting algorithmic implications. It is possible to compute highest flooding using a minimum spanning tree of the graph with a dramatically lower number of edges. However, one has to take in the balance the time needed for constructing the graph. It may be interesting if one has to construct several dominated flooding of the same graph. We will meet later an algorithm which combines the construction of the MST and of a particular flooding of the graph. 


\section{Flooding with shortest distance algorithms}

Many shortest distance algorithms exist. Each of the following has specific advantages.

Algorithm of Berge

Algorithm of Dijkstra

Algorithm of Prim

Core expanding algorithm

Path algebra 
Algorithms for computing the highest flooding on tank networks 


\section{The algorithm of Berge}




\section{The algorithm of Berge}

\section{Initialisation}

The algorithm of Berge is initialised with the function $\omega$. For each node $p: \tau_{p}=\omega_{p}$.

This distribution being not a flooding, the algorithm applies until stability the relation Repeat until $\tau_{p}^{(m)}=\tau_{p}^{(m-1)}: \tau_{p}^{(n)}=\omega_{p} \wedge \bigwedge_{q \text { neighbor of } p}\left(\tau_{q}^{(n-1)} \vee e_{p q}\right)$

Convergence: $\tau_{p}^{(m)}$ decreases at each iteration. It has a lower ceiling, the smallest value of $\omega$, therefore it converges. 


\section{The algorithm of Berge}

\section{Improved version with less memory accesses:}

As $\tau$ can only decrease at each iteration, replacing the ceiling function $\omega$ by the value taken by $\tau$ at iteration $(n-1)$ produces an equivalent algorithm with less memory accesses: the value of $\omega$ has only to be fetched at initialization.

Initialisation: $\tau_{p}^{(0)}=\omega_{p}$

Repeat until $\tau_{p}^{(m)}=\tau_{p}^{(m-1)}: \tau_{p}^{(n)}=\tau_{p}^{(n-1)} \wedge \wedge_{q \text { neighbor of } p}\left(\tau_{q}^{(n-1)} \vee e_{p q}\right)$ 


\section{Software or hardware implementation of the algorithm of}

\section{Berge}

Using a local neighborhood, extremely versatile, as the nodes may be processed in any order, the algorithm of Berge $t$ is well suited for software or hardware implementation based on a systematic scan of the graph. The algorithm is parallel or recursive:

- $\tau_{p}^{(n)}=\tau_{p}^{(n-1)} \wedge \bigwedge_{q \text { neighbor of } p}\left(\tau_{q}^{(n-1)} \vee e_{p q}\right)$ represents a parallel implementation of the algorithm : the arguments for computing $\tau_{p}^{(n)}$ are all those obtained during the previous scan.

- The recursive implementation separates the nodes already met during the current scanning and the nodes in the future:

$\tau_{p}^{(n)}=\tau_{p}^{(n-1)} \wedge \bigwedge_{q \text { past neighbor of } p}\left(\tau_{q}^{(n)} \vee e_{p q}\right) \wedge$

$q$ future neighbor of $p$

$$
\bigwedge_{\text {neighbor of } p}\left(\tau_{q}^{(n-1)} \vee e_{p q}\right)
$$

Alternating a forward scan and a backward scan for the graph permits

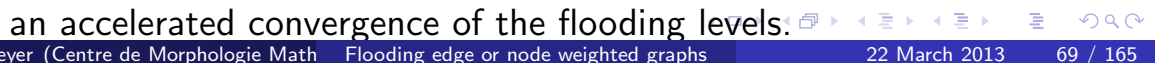


The algorithm of Moore-Dijkstra 


\section{The Moore Dijkstra algorithm}

The Dijkstra algorithm is a greedy algorithm. A set $S$ contains all nodes whose distance is known. For the outside neighbors of $S$, this distance is estimated: for $p \in S$ and $q \notin S, \tau_{q} \leq \tau_{p} \vee e_{p q}$. And we have $\tau_{q}=\tau_{p} \vee e_{p q}$ if the shortest path to $q$ follows the edge $(p, q)$. This is the case for the node in $\bar{S}$ with the lowest estimation. This node may be introduced into $S$ and the estimation of the distance of its neighbors still in $\bar{S}$ updated. The nodes introduced in $S$ have increasing values, as the estimation of all nodes in $\bar{S}$ is higher than the estimation of the nodes in $S$.

The edges linking each node with the node through which it has been flooded in the algorithm form a tree. This tree is rooted at $\Omega$ and contains a never decreasing geodesic path between $\Omega$ and each node. 


\section{The Moore Dijkstra algorithm}

\section{Initialisation:}

$$
S=\{\Omega\} \text { and } \tau_{\Omega}=-\infty ; \text { for each node } p \text { in } N=\bar{S}: \tau_{p}=\omega_{p}
$$

\section{Flooding:}

While $\bar{S} \neq \varnothing$ repeat:

Select $j \in \bar{S}$ for which $\tau_{j}=\min _{i \in \bar{S}}\left[\tau_{i}\right]$

$\bar{S}=\bar{S} \backslash\{j\}$

For any neighbor $i$ of $j$ in $\bar{S}$ do $\tau_{i}=\min \left[\tau_{i}, \tau_{j} \vee e_{j i}\right]$

End While 
The Moore Dijkstra algorithm without the dummy node and edges

The dummy node $\Omega$ and the dummy edges linking $\Omega$ with the nodes of $N$ is useless in practice:

\section{Initialization:}

$$
S=\varnothing ; \bar{S}=N ; \text { for each node } p \text { in } N: \tau_{p}=\omega_{p}
$$

\section{Flooding:}

While $\bar{S} \neq \varnothing$ repeat:

Select $j \in \bar{S}$ for which $\tau_{j}=\min _{i \in \bar{S}}\left[\tau_{i}\right]$

$\bar{S}=\bar{S} \backslash\{j\}$

For any neighbor $i$ of $j$ in $\bar{S}$ do $\tau_{i}=\min \left[\tau_{i}, \tau_{j} \vee e_{j i}\right]$

End While 


\section{Simplification of the algorithm of Dijkstra}

When the node $j$ is introduced into $S$, it has the highest value in $S$. The instruction

$<$ For any neighbor $i$ of $j$ in $\bar{S}$ do $\tau_{i}=\min \left[\tau_{i}, \tau_{j} \vee e_{j i}\right]>$

may be simplified in:

$<$ For any neighbor $i$ of $j$ verifying $\tau_{j} \vee e_{j i}<\tau_{i}$ do $\tau_{i}=\tau_{j} \vee e_{j i}>$ as a node $i$ verifying $\tau_{i}>\tau_{j}$ cannot belong to $S$. Checking whether $i$ belongs to $\bar{S}$ is not necessary, leading to the following algorithm. 


\section{Simplification of the algorithm of Dijkstra}

Initialization:

$$
S=\varnothing ; \bar{S}=N ; \text { for each node } p \text { in } N: \tau_{p}=\omega_{p}
$$

Flooding:

While $\bar{S} \neq \varnothing$ repeat:

Select $j \in \bar{S}$ for which $\tau_{j}=\min _{i \in \bar{S}}\left[\tau_{i}\right]$

$\bar{S}=\bar{S} \backslash\{j\}$

For any neighbor $i$ of $j$ verifying $\tau_{j} \vee e_{j i}<\tau_{i}$ do $\tau_{i}=\tau_{j} \vee e_{j i}$

End While 


\section{The algorithm of Dijkstra with a funnel structure}

Fetching the node with the smallest value $\tau_{j}$ within $\bar{S}$ is made easy by using an adequate data structure such as a "funnel" structure: nodes with any order of priority may be stored in the funnel; but only one of the nodes with the smallest priority is extracted at any time. Possible implementation: an ordered bucket structure. A node is introduced in the bucket corresponding to its priority. Each extracted node is chosen among the nodes in the bucket with highest priority. If the buckets have the structure of a queue, we speak about hierarchical queues. 


\section{The algorithm of Dijkstra with a funnel structure}

Before being introduced into $S$, the distance of each node has to be estimated anew every time one of its neighbor is introduced into $S$. If we use a "singel occupancy funnel", a node occupies only one location and its priority is updated if needed. In a multiple occupancy funnel (MOF), a node may occupy more than one location, with distinct priority. When a node is extracted for the first time, its value is correct. When it is extracted another time one has to discard it. 


\section{The algorithm of Dijkstra with a funnel structure}

Consider a flooding of the following graph. Initially the node $p$ is in $\Phi$ with a value 3 and a yellow colour. The two other nodes are not in $\Phi$. When $p$ is extracted from $\Phi$, both its neighbors are introduced in $\Phi$ with estimates equal to 5 and 9 (yellow colour). The node $q$ is then extracted from $\Phi$ and its neighbor $r$ is introduced in $\Phi$ with a new estimate 7.; at the same time, the node $r$ of the graph gets this same value. When $r$ is extracted from $\Phi$ for the first time, there is an identity between its priority and the flooding level of $r$ in the graph. The second time it is extracted, its priority is 9 , higher than the value in the graph and has to be discarded from further processing.
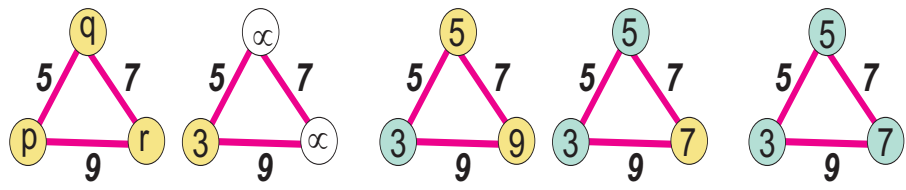

Figure: Propagation of the flooding on an edge weighted graph using the Dijkstra algorithm.

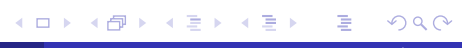




\section{The algorithm of Dijkstra with a funnel structure}

\section{Initialization:}

create $\Phi$, a multi occupancy funnel ; $\tau=\infty$

for each node $p$ verifying $\omega_{p}<\infty$ introduce $p$ into $\Phi$ with a priority $\omega_{p} ; \tau_{p}=\omega_{p}$

\section{Flooding}

While $\Phi$ is not empty repeat:

Extract from $\Phi$ the node $j$ with the lowest prioriy $\lambda$

$$
\text { If } \tau_{j}=\lambda
$$

For any neighbor $i$ of $j$ such that $\tau_{j} \vee e_{j i}<\tau_{i}$

$$
\begin{aligned}
& \tau_{i}=\tau_{j} \vee e_{j i} \\
& \text { introduce } i \text { into } \Phi \text { with the priority } \tau_{i}
\end{aligned}
$$

End While 


\section{Marker based segmentation}

In the case of marker based segmentation a number of nodes are markers. The aim is to produce a Voronoi tessellation of the graph: each node is assigned to the marker which is closest for the ultrametric distance. Several solutions exist for breaking the ties, if a node is at the same distance of distinct markers.

The preceding algorithm may be used, the reduced set $\widehat{S}$ of ceiling minima, being the set of nodes belonging to the markers. In the context of segmentation, a distinct label is assigned to each marker. This label will be assigned to the total region flooded through this marker. 


\section{Marker based segmentation}

\section{Initialization:}

create a $\mathrm{HQ} \Phi$

create an image $\zeta$ which will hold the labels

assign to each marker node $p$ a distinct label $\zeta_{p}$, a flooding value $\tau_{p}=0$ and introduce $p$ into $\Phi$ with a priority 0 for all other nodes $\tau=\infty$

\section{Flooding}

While $\Phi$ is not empty repeat:

Extract from $\Phi$ the node $j$ with the lowest prioriy $\lambda$

$$
\text { If } \tau_{i}=\lambda
$$

For any neighbor $i$ of $j$ such that $\tau_{j} \vee e_{j i}<\tau_{i}$

$$
\begin{aligned}
& \tau_{i}=\tau_{j} \vee e_{j i} \\
& \zeta_{i}=\zeta_{j} \\
& \text { introduce } i \text { into } \Phi \text { with the priority } \tau_{i}
\end{aligned}
$$

End While 


\section{Advantages of a hierarchical queue}

A hierarchical queue are governed by a double hierarchical order. A hierarchical queue is a series of first first out queues, having each a priority. A node is introduced in the queue corresponding to its priority. The node which is extracted at any time of the $\mathrm{HQ}$ is the node which has been introduced first in the queue with the highest priority. The priorities among the queues organize that the flooding progresses in an order of increasing altitudes. The FIFO structure of the queue ensures that a node inside a plateau is flooded in an order proportional to its distance to the lower border of the plateau. 


\section{The algorithm of Prim}




\section{Constructing the MST}

On a graph, from a node $p$ to a node $q$, the flood always follows the paths of lowest sup-section linking $p$ and $q$. All such paths belong to the MST of the graph. Hence, it is possible to combine the flooding with the construction of the MST. The algorithm of PRIM constructs the MST rooted in $\Omega$.

\section{Initialisation}

Initially, the tree $T$ spans only the node $\Omega$.

\section{Expansion}

As long as the tree does not contain all nodes of the graph:

Chose the lowest edge $(q, s)$ in the cocycle of $T$, such that $q \in T$ and $s \notin T$. Append the node $s$ to the tree: $T=T \cup\{s\}$ 


\section{Flooding an edge weighted graph following its MST}

Flooding with the algorithm of PRIM

The result of the preceding algorithm is a tree rooted at $\Omega$. Each other node $p$ is linked with $\Omega$ through a unique path. The flood coming from $\Omega$ necessarily follows this path. The flooding of the nodes and the construction of the tree may be done simultaneously.

\section{Initialisation}

Initially, the tree $T$ spans only the node $\Omega: T=\{\Omega\} . \tau_{\Omega}=0$.

\section{Expansion}

As long the tree does not contain all nodes of the graph:

Chose the lowest edge $(q, s)$ in the cocycle of $T$, such that $q \in T$ and $s \notin T$.

Append the edge $(q, s)$ and the node $s$ to the tree: $T=T \cup\{s\}$ $\tau_{s}=\tau_{q} \vee e_{q s}$ 


\section{Flooding an edge weighted graph following its MST}

\section{Analysis of the algorithm}

The nodes are introduced with a never decreasing flood level. A node with

a flood level $\lambda$ first floods its neighbors appended through an edge which is lower or equal to the current flooding level: these neighbors get the current flood level $\lambda$ and are appended to the tree. If this is not possible anymode, the smallest edge in the cocycle of the tree with a weight $>\lambda$ is followed, introducing the first node with a weight $>\lambda$ into the tree.

The PRIM algorithm is a particular avatar of Dijkstra's algorithm. Among all neighboring nodes of $T$ for which the estimated flooding level is the smallest, the algorithm of PRIM first considers those linked with the tree through the lowest edge. 


\section{Scheduling with a $\mathrm{HQ}$}

\section{Initialization:}

create $\Phi$, a multi occupancy funnel.

$\tau=\infty$

for each node $p$ verifying $\omega_{p}<\infty$, introduce $p$ into $\Phi$ with a priority $\omega_{p}$

$$
\lambda=-\infty
$$

\section{Flooding}

While $\Phi$ is not empty repeat:

Extract from $\Phi$ the node $p$ with the lowest prioriy $\mu$

$$
\begin{aligned}
& \text { if } \tau_{p}=\infty \\
& \text { If } \mu>\lambda: \lambda=\mu \\
& \tau_{p}=\lambda
\end{aligned}
$$

For any neighbor $q$ of $p$ such that $\tau_{q}=\infty$ introduce $q$ into $\Phi$ with the priority $e_{p q}$

Remark: Replacing the last instruction with <introduce $i$ into the funnel with the priority $\lambda \vee e_{j i}>$ produces the algorithm of Dijkstra. 


\section{Marker based segmentation with the algorithm of Prim}

\section{Initialisation}

$T=\{\varnothing\}$

For each marker $p$ :

$$
\begin{aligned}
& \tau_{p}=0 \\
& T=T \cup\{p\} \\
& \text { assign a new label } \zeta_{p}
\end{aligned}
$$

\section{Expansion}

As long the tree does not contain all nodes of the graph:

Chose the lowest edge $(q, s)$ in the cocycle of $T$, such that $q \in T$ and $s \notin T$.

Append the edge $(q, s)$ and the node $s$ to the tree: $T=T \cup\{s\}$

$$
\begin{aligned}
& \tau_{s}=\tau_{q} \vee e_{q s} \\
& \zeta_{s}=\zeta_{q}
\end{aligned}
$$




\section{Marker based segmentation with the algorithm of Prim}

If we are not interested by the flooding level but only by the Voronoi partition associated to the markers:

\section{Initialisation}

For each marker $p$, assign a new label $\zeta_{p}$

For all other nodes $q: \zeta_{q}=-\infty$

\section{Expansion}

As long as there are nodes with a label $\zeta=-\infty$

Chose the lowest edge $(q, s)$ verifying $\zeta_{q}>-\infty$ and $\zeta_{s}=-\infty$ $\zeta_{s}=\zeta_{q}$ 


\section{Shortest path algorithms on node weighted graphs}




\section{From edge weighted floodings to node weighted floodings}

Any flooding $\tau$ of a node weighted graph $G_{n}$, above the ground level $f$ also is a flooding on an edge weighted graph $G_{e}$ with edge weights $e_{p q}=f_{p} \vee f_{q}:->$ all results and algorithms established for $G_{e}$ are applicable to $r G_{n}$ simply by replacing $e_{p q}$ by $f_{p} \vee f_{q}$ and remembering that $\tau \geq f$, they get simpler. 


\section{The algorithm of Berge}




\section{The algorithm of Berge}

Initialisation: $\tau_{p}^{(0)}=\omega_{p}$

Repeat until $\tau_{p}^{(m)}=\tau_{p}^{(m-1)}: \tau_{p}^{(n)}=$

$\omega_{p} \wedge \bigwedge_{q \text { neighbor of } p}\left(\tau_{q}^{(n-1)} \vee f_{p} \vee f_{q}\right)=\omega_{p} \wedge \bigwedge_{q \text { neighbor of } p}\left(\tau_{q}^{(n-1)} \vee f_{p}\right)$

\section{A variant of the algorithm}

Replacing the ceiling function $\omega$ by the value taken by $\tau$ at iteration $(n-1)$ since $\tau$ can only decrease at each iteration. The algorithm becomes:

Initialisation: $\tau_{p}^{(0)}=\omega_{p}$

Repeat until $\tau_{p}^{(m)}=\tau_{p}^{(m-1)}: \tau_{p}^{(n)}=\tau_{p}^{(n-1)} \wedge \bigwedge_{q \text { neighbor of } p}\left(\tau_{q}^{(n-1)} \vee f_{p}\right)$

We recognize the classical algorithm. Repeat until stability $\tau=f \vee \varepsilon \tau$ 


\section{The algorithm of Prim}




\section{The algorithm of Prim}

The algorithm of PRIM remains exactly the same as for pipe networks. By replacing $e_{q s}$ by its value $f_{q} \vee f_{s}$, and since $\tau_{q} \geq f_{q}$, we get $\tau_{q} \vee f_{q} \vee f_{s}=\tau_{q} \vee f_{s}$. The flooding of the nodes and the construction of the tree may be done simultaneously.

\section{Initialisation}

Initially, the tree $T$ has only the node $\Omega$ and no edge:

$T=\{\Omega, \varnothing\} . \tau_{\Omega}=0$.

\section{Expansion}

As long the tree does not contain all nodes of the graph:

Chose the edge $(q, s)$ with the lowest weight $f_{q} \vee f_{s}$ in the cocycle of $T$, such that $q \in T$ and $s \notin T$.

Assign the node $s$ to the tree: $T=T \cup\{s\}$

$$
\tau_{s}=\tau_{q} \vee f_{q} \vee f_{s}=\tau_{q} \vee f_{s}
$$


The algorithm of Dijkstra 


\section{The algorithm of Dijkstra}

Initialization:

$$
S=\varnothing ; \text { for each node } p \text { in } N: \tau_{p}=\omega_{p}
$$

Flooding:

While $S \neq N$ repeat:

Select $j \in \bar{S}$ for which $\tau_{j}=\min _{i \in \bar{S}}\left[\tau_{i}\right]$

$S=S \cup\{j\}$

For any neighbor $i$ of $j$ verifying $\tau_{i}>\tau_{j} \vee f_{i}$ do $\tau_{i}=\tau_{j} \vee f_{i}$

End While 


\section{Avoiding unnecessary work}

$j$ is the smallest neighbor of $i$. If $i$ is flooded through one of its neighbors, this neighbor can only be $j$ and as soon the value $\tau_{i}$ is computed once, this value is correct and final : $\tau_{i}=\tau_{j} \vee f_{i}$. For this reason, we may use an image of binary flags $\zeta$, in order to flag all nodes for which the flooding value is known Initialization:

$$
S=\varnothing ; \text { for each node } p \text { in } N: \tau_{p}=\omega_{p} \text { and } \zeta_{p}=0
$$

Flooding:

While $S \neq N$ repeat:

Select $j \in \bar{S}$ for which $\tau_{j}=\min _{i \in \bar{S}}\left[\tau_{i}\right]$

$S=S \cup\{j\}$

For any neighbor $i$ of $j$ such that $\zeta_{i}=0$ and $\tau_{i}>\tau_{j} \vee f_{i}$

$$
\begin{aligned}
& \zeta_{i}=1 \\
& \tau_{i}=\tau_{j} \vee f_{i}
\end{aligned}
$$

End While

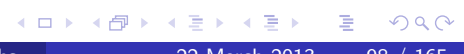


The regional minima of the ceiling function are sufficient, and any overset of them... 


\section{Finding an overset of the regional minima nodes of the ceiling function}

Each regional minimum of a flooding contains a regional minimum of the ceiling function $\omega$. If it were not constrained at this level, the level of the flooding would be higher in this regional minimum. Outside the regional minima, the level of the flooding not constrained by $\omega$.

Ideally, the algorithm of Dijkstra should be initialized using one node and only one node in each regional minimum of $\omega$. As the regional minima are costly to compute, a cheap overset of these nodes offers a better compromise. 


\section{A first overset of the ceiling minima}

The regional minima of $\omega$ are plateaus of uniform altitude, without lower neighbors. During a forward raster scan of the image, the pixels are detected which have only higher neighbors in the past and no lower neighbor in the future. The set $X$ is obained in a forward scan through the image (for a 2D image, from left to rights and from top to bottom).

$X=\left\{p \mid \tau_{p}<\bigwedge_{q \in \operatorname{past}(p)} \tau_{q}\right\} \wedge\left\{p \mid \tau_{p} \leq \bigwedge_{q \in \text { future }(p)} \tau_{q}\right\}$

This algorithm finds the entry points in the regional minima and in a certain number of plateaus. We may reduce the plateaus as follows. 


\section{Reducing the number of candidates}

A classical algorithm for constructing regional minima. The flooding of $\omega$ with $\omega+1$ as ceiling function produces a new function $\widehat{\omega}$. The regional minima of $\omega$ are all nodes verifying $\widehat{\omega}$. For the sake of economy only a partial flooding is done, suppressing a number of plateaus which are not regional minima:

- using geodesic erosions defined as:

$\varepsilon_{\omega}(g)=\varepsilon g \vee \omega$

$\varepsilon_{\omega}^{(n)}(g)=\varepsilon_{\omega}\left(\varepsilon_{\omega}^{(n)}(g)\right)$

The set of nodes $Y$ verifying $\varepsilon_{\omega}^{(n)}(g)>\omega$ is an overset of the regional minima, decreasing with the number of iterations $n$.

- Using one pass of a recursive geodesic erosion of $\omega$ above $g$ with a backward scanning order $\overleftarrow{\varepsilon} \omega(g)(p)=\omega_{p} \vee \bigwedge_{q \in \operatorname{past}(p)} g_{q}$

The set $Z$ verifying $\overleftarrow{\varepsilon} \omega(g)>\omega$ is an overset of the regional minima

CEILING_MINIMA is one of the following sets : 


\section{The Dijkstra algorithm with a reduced initialisation set of} ceiling minima.

A binary tag $\zeta: \zeta=0$ for nodes with an unknown flooding level; $\zeta=1$ after the first time the flooding level is computed.

\section{Initialization:}

create a $\mathrm{HQ} \Phi ; \tau=\infty ; \zeta=0$

for each node $p$ belonging to $\widehat{S}=$ " ceiling minima",

introduce $p$ into $\Phi$ with a priority $\omega_{p}$

$$
\tau_{p}=\omega_{p}
$$

\section{Flooding}

While $\Phi$ is not empty repeat:

Extract from $\Phi$ the node $j$ with the lowest prioriy $\lambda$

$$
\text { If } \tau_{i}=\lambda
$$

For any neighbor $i$ of $j$ such that $\zeta_{i}=0$ and $\tau_{j} \vee e_{j i}<\tau_{i}$

$$
\tau_{i}=\tau_{j} \vee e_{j i} ; \zeta_{i}=1 \text {; introduce } i \text { into } \Phi \text { with the }
$$

priority $\tau_{i}$

End While 


\section{An equivalent algorithm with a 3 state tag}

The following algorithm is equivalent and uses a 3 state flag: ("unknown", "final", "in $\left.S^{\prime \prime}\right)=(" u$ ", " $f$ ", " $s ")=(0,1,2)$ with the following meanings: a) "unknown" $=" u$ " $=0$ are the nodes whose flooding value has not been computed yet; b) "final" =" $f$ " $=1$ computed nodes, not yet in $S$; c) "in $S^{\prime \prime}=" s^{\prime \prime}=2$ for pixels introduced into $S$ 


\section{Flooding algorithm}

\section{Initialization:}

create a $\mathrm{HQ} \Phi ; \tau=\infty ; \zeta=0$

for each node $p$ belonging to $\widehat{S}=$ "ceiling minima",

introduce $p$ into $\Phi$ with a priority $\omega_{p}$

$$
\tau_{p}=\omega_{p}
$$

\section{Flooding}

While $\Phi$ is not empty repeat:

Extract from $\Phi$ the node $j$ with the lowest prioriy

$$
\text { if } \begin{aligned}
\zeta_{j}<2 \\
\zeta_{j}=2
\end{aligned}
$$

For any neighbor $i$ of $j$ such that $\zeta_{i}=0$

$$
\begin{aligned}
& \text { if } \tau_{j} \vee e_{j i}<\tau_{i} \text { then } \tau_{i}=\tau_{j} \vee e_{j i} \\
& \zeta_{i}=1 \text {; introduce } i \text { into } \Phi \text { with the priority } \tau_{i}
\end{aligned}
$$

End While 


\section{Illustration}

The figure presents in red a topographic surface and in green a ceiling function. The successive lines below the figure present the evolution of the algorithm. The algorithm is illustrated step by step. In each group of 3 lines

- the first represent the status of the node, "u"=unknow, " $f^{\prime}=$ flooded, " $s$ " in S

- the second represent the current estimated flooding value of the nodes

- the third represent the nodes in $\Phi$ 


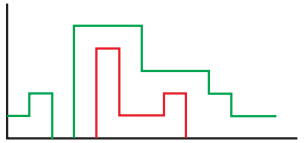

u u u u u u u u u u u u 999999999999 \# \# 0 \#\#\# 3 \#\#\# 1 \# u f $s$ f u u u u u u u 9000009999999999 \# 0 \# 0 \#\# 3 \#\# \# 1 \#

f $s$ s f u u u u u u u 0000099999999 0 \# \# 0 \#\# 3 \#\#\# 1 \# s s $s$ f u u u u u u u 00000999999999 \#\# \# 0 \#\# 3 \# \# \# 1 \# s s s s f u u u u u u u 0000049999999 \#\# \# \# 4 \# 3 \# \# \# 1 \# s s s s f u u u f $\mathrm{s} f$ $\begin{array}{llllllllllll}0 & 0 & 0 & 0 & 4 & 9 & 9 & 9 & 9 & 1 & 1 & 1\end{array}$ \# \# \# \# 4 \# 3 \#\# 1 \# 1 s s s s f u u u u f s s $\begin{array}{llllllllllll}0 & 0 & 0 & 0 & 4 & 9 & 9 & 9 & 9 & 1 & 1 & 1\end{array}$ \# \# \# \# \# 3 \#\# 1 \# \# s s s s f u u u f s s $\begin{array}{llllllllllll}0 & 0 & 0 & 0 & 4 & 9 & 9 & 9 & 1 & 1 & 1 & 1\end{array}$ \# \# \# 4 \# 3 \# 1 \# \# \# s s s f $\mathrm{f} u$ f $\mathrm{s}$ s $\mathbf{s}$ s

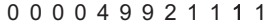
\#\# \#\# 4 \# 32 \# \# \# \# s s s s f u f s s s s s $\begin{array}{llllllllllll}0 & 0 & 0 & 0 & 4 & 9 & 2 & 2 & 1 & 1 & 1 & 1\end{array}$ \# \# \# \# 4 \# 2 \# \# \# \# \# s s s s f f s s s s s $\begin{array}{lllllllllll}0 & 0 & 0 & 0 & 4 & 2 & 2 & 2 & 1 & 1 & 1\end{array}$ \# \# \# \# 42 \# \# \# \# \# \# s s s $\mathbf{s}$ f $s$ s s s s $\begin{array}{lllllllllll}0 & 0 & 0 & 0 & 4 & 2 & 2 & 2 & 1 & 1 & 1\end{array}$ \# \# \# \# 4 \# \# \# \# \# \# s s s s s s s s s s $s$ $\begin{array}{lllllllllll}0 & 0 & 0 & 0 & 4 & 2 & 2 & 2 & 1 & 1 & 1\end{array}$ \# \# \# \# \# \# \# \# \# \# \# \#

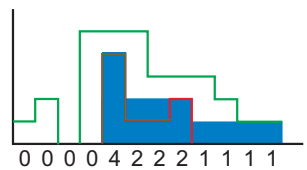




\section{Speeding up the flooding}

In the Dijkstra algorithm, $S$ contains all nodes with a known flooding; at each iteration, the node with the smallest estimated flooding value is introduced into $S$. One node is introduced at each iteration of the algorithm. Faster floodings are possible if one remarks:

(1) if $f_{p}=f_{q}$, then $\tau_{p}=\tau_{q}$, as the flooding is a connected operator.

(2) If $f_{p}=\omega_{p}$, then ground and ceiling levels are identical, hence $\tau_{p}=f_{p}=\omega_{p}$

(3) if $f_{p} \vee f_{q}<\tau_{p}$ then $\tau_{q}=f_{q}$. The proof is the following. $\left\{f_{p} \vee f_{q}<\tau_{p}\right\} \Leftrightarrow\left\{f_{p}<\tau_{p}\right.$ and $\left.f_{q}<\tau_{p}\right\}$. On the other hand the criterion for floodings $\left\{\tau_{p}>\tau_{q}\right\} \Rightarrow\left\{f_{p}=\tau_{p}\right\}$ is equivalent with $\left\{f_{p}<\tau_{p}\right\} \Rightarrow\left\{\tau_{p} \leq \tau_{q}\right\}$. On the other hand $\tau_{q} \leq \tau_{p} \vee f_{q}=\tau_{p} \vee f_{p} \vee f_{q}=\tau_{p}$ which shows that $\tau_{p}=\tau_{q}$

(4) if $f_{q}<\tau_{p}$ then $\tau_{q} \leq f_{q} \vee \tau_{p}=\tau_{p}$. . 


\section{Speeding up the algorithm of Dijkstra}

The two following rules permit to speed up the algorithm of Dijkstra.

(1) if $f_{q} \geq \tau_{p}$ then $\tau_{q}=f_{q}$. The proof is the following. For any neighboring nodes $p$ and $q$ the flooding levels verify $\tau_{q} \leq \tau_{p} \vee f_{q}=f_{q}$. But as $\tau_{q} \geq f_{q}$ we get $\tau_{q}=f_{q}$.

(2) suppose that $(p, q)$ are neighbors and $p$ is the node of $\partial^{-} S$ for which the flooding level $\tau_{p}$ is the lowest. If $f_{q}<\tau_{p}$, and if $q$ is to be flooded by a node in $S$, this node necessarily is $p$ and the estimated flooding level of $q$ is $\tau_{q}=\tau_{p} \vee f_{q}=\tau_{p}$. 


\section{The core expanding algorithm}

We may now derive a fast algorithm from these remarks. Suppose that during the flooding, the set $S$ represents all flooded nodes and $p$ is the node of $\partial^{-} S$ for which the flooding level $\tau_{p}$ is the lowest. If $f_{q} \geq \tau_{p}$ then $\tau_{q}=f_{q}$. If on the contrary $f_{q}<\tau_{p}$, we apply Dijkstra's algorithm and introduce into $S$ the node with the smallest estimate. If there exists in $\bar{S}$ a node $j$ with a smaller ceiling value $\omega_{j}$ as $\tau_{p}: S=S \cup\{j\}$. If not, $\tau_{q}=\tau_{p}$ is the flooding estimation of a node in $\bar{S}$ which is the lowest and $S=S \cup\{q\}$.

This shows that in the case where $\omega_{j} \geq \tau_{p}$, all neighbors of $p$ may be introduced at once into the set $S$, yielding the following algorithm: 


\section{The core expanding algorithm}

\section{Initialization:}

$$
S=\varnothing ;
$$

\section{Flooding:}

While $S \neq N$ repeat:

Set $\lambda=\min _{i \in \bar{S}}\left[\omega_{i}\right]$; if $j$ does not exist $\lambda=\infty$

Set $\mu=\tau_{p}$ for $p \in \partial^{-} S$ for which $\tau_{p}=\min _{i \in \partial^{-} S}\left[\tau_{i}\right]$

$$
\text { if } \lambda<\mu: S=S \cup\{j\} \text { and } \tau_{j}=\omega_{j}
$$

else

For each neighbor $q$ of $p$ in $\bar{S}$ do:

End While

$$
\begin{aligned}
& \tau_{q}=\tau_{p} \vee f_{q} \\
& S=S \cup\{q\}
\end{aligned}
$$

At each iteration, the algorithm has to fetch the node $p$ with the smallest flooding value in $\partial^{-} S$ which is easily done with a $\mathrm{HQ}$; the node $j$ with the smallest ceiling value $\omega_{j}$. can be easily done if the nodes are ordered with increasing values in a FIFO. 


\section{Illustration of the core expanding algorithm}

The topographic surface is in red and the ceiling function in green. At initialisation, the nodes with a label " $i$ " are the nodes of the ceiling minima. The smallest of them is introduced into $S$ and immediately expanded, introducing both its neighbors into $S$. In line 5 , another ceiling minima is introduced into $S$ and immediately expanded. 


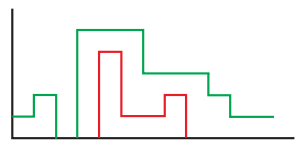

u u(i) u u i u u u i u 120555333211 u u(s) u u u i u u u i u 100055333211 u(s)s s u u i u u u i u 000055333211 s s s(s) $\mathbf{s} u$ i u u u i u 000045333211 s s s s s u i u us(s) $0 \begin{array}{lllllllllll}0 & 0 & 0 & 4 & 5 & 3 & 3 & 3 & 1 & 1 & 1\end{array}$ s s s s s u i u s(s) s $\begin{array}{llllllllllll}0 & 0 & 0 & 0 & 4 & 5 & 3 & 3 & 1 & 1 & 1 & 1\end{array}$ s s s s s u i s s s s $0 \begin{array}{llllllllll}0 & 0 & 0 & 4 & 5 & 2 & 1 & 1 & 1\end{array}$ s s s s us s s s 000045221111 s s s s s s s s s s 00000422201111

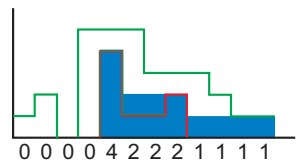

Figure: expanding algorithm 


\section{The lakes of an ultrametric distance function form a dendrogram}




\section{Distance on a graph based on the maximal edge weight}

along the chain

The weights are assigned to the edges, and represent their altitudes. Altitude of a chain: The altitude of a chain is equal to the highest weight of the edges along the chain.

Flooding distance between two nodes: The flooding distance fldist $(x, y)$ between nodes $x$ and $y$ is equal to the minimal altitude of all chains between $x$ and $y$. During a flooding process, in which a source is placed at location $x$, the flood would proceed along this chain of minimal highest altitude to reach the pixel $y$. If there is no chain between them, the level distance is equal to $\infty$.

Triangular inequality : For $(x, y, z): d(x, z) \leq d(x, y) \vee d(y, z)$ : ultrametric inequality 


\section{The flooding distance is an ultrametric distance}

An ultrametric distance verifies

$*$ reflexivity: $d(x, x)=0$

* symmetry: $d(x, y)=d(y, x)$

* ultrametric inequality: for all $x, y, z: d(x, y) \leq \max \{d(x, z), d(z, y)\}$ : the lowest lake containing both $x$ and $y$ is lower or equal than the lowest lake containing $x, y$ and $z$. 


\section{The balls of an ultrametric distance}

For $p \in E$ the closed ball of centre $p$ and radius $\rho$ is defined by $\operatorname{Ball}(p, \rho)=\{q \in E \mid d(p, q) \leq \rho\}$. The open ball of centre $p$ and radius $\rho$ is defined by $\operatorname{Ball}(p, \rho)=\{q \in E \mid d(p, q)<\rho\}$.

Every triangle is isosceles. Let us consider three distinct points $p, q, r$ and suppose that the largest edge of this triangle is $p q$. Then $d(p, q) \leq d(p, r) \vee d(r, q)$, showing that the two larges edges of the triangle have the same length.

\section{Lemma}

Each element of a closed ball $\operatorname{Ball}(p, \rho)$ is centre of this ball

Proof: Suppose that $q$ is an element of $\operatorname{Ball}(p, \rho)$. Let us show that then $q$ also is centre of this ball. If $r \in \operatorname{Ball}(p, \rho)$ :

$d(q, r) \leq \max \{d(q, p), d(p, r)\}=\rho$, hence $r \in \operatorname{Ball}(q, \rho)$, showing that $\operatorname{Ball}(p, \rho) \subset \operatorname{Ball}(q, \rho)$. Exchanging the roles of $p$ and $q$ shows that $\operatorname{Ball}(p, \rho)=\operatorname{Ball}(q, \rho)$ 


\section{The balls of an ultrametric distance}

\section{Lemma}

Two closed balls Ball $(p, \rho)$ and $\operatorname{Ball}(q, \rho)$ with the same radius are either disjoint or identical.

Proof: If $\operatorname{Ball}(p, \rho)$ and $\operatorname{Ball}(q, \rho)$ are not disjoint, then they contain at least one common point $r$. According to the preceding lemma, $r$ is then centre of both balls $\operatorname{Ball}(p, \rho)$ and $\operatorname{Ball}(q, \rho)$, showing that they are identical.

\section{Lemma}

The radius of a ball is equal to its diameter.

Proof: Let Ball $(p, \rho)$ be a ball of radius $\rho$. Let $q$ and $r$ be two nodes with the largest distance in $\operatorname{Ball}(p, \rho)$. This distance $\lambda$ is called diameter of the ball and verifies : $\lambda=d(q, r) \leq d(q, p) \vee d(p, r) \leq \rho$. Hence $\lambda \leq \rho$. If there exists two nodes in $\operatorname{Ball}(p, \rho)$ with a distance equal to $\rho$, then $\lambda \geq \rho$. In this case $\lambda=\rho$. 


\section{Reminders on dendrograms}




\section{The structure associated to an order relation}

$E$ : a domain with a finite number of elements called points $\mathcal{X}$ : a subset of $\mathcal{P}(E)$, with the order relation $\subset$ $\operatorname{supp}(\mathcal{X})$ : union of all sets belonging to $\mathcal{X}$ is called support of $\mathcal{X}$ : $\operatorname{supp}(\mathcal{X})$.

The subsets of $\mathcal{X}$ may be structured into: 
- the summits: $\operatorname{Sum}(\mathcal{X})=\{A \in \mathcal{X} \mid \forall B \in \mathcal{X}: A \subset B \Rightarrow A=B\}$

- the leaves: $\operatorname{Leav}(\mathcal{X})=\{A \in \mathcal{X} \mid \forall B \in \mathcal{X}: B \subset A \Rightarrow A=B\}$

- the predecessors: $\operatorname{Pred}(A)=\{B \in \mathcal{X} \mid A \subset B\}$

- the immediate predecessors : $\operatorname{ImPred}(A)=\{B \in \mathcal{X} \mid\{U \mid U \in \mathcal{X}, A \subset U$ and $U \subset B\}=(A, B)\}$

- the successors: $\operatorname{Succ}(A)=\{B \in \mathcal{X} \mid B \subset A\}$

- the immediate successors : $\operatorname{ImSucc}(A)=\{B \in \mathcal{X} \mid\{U \mid U \in \mathcal{X}, B \subset U$ and $U \subset A\}=(A, B)\}$

- the uncles: uncle $(A)=\{B \in \mathcal{X} \mid \operatorname{ImPred}(B) \in \operatorname{Pred}(A), B \notin$ $\operatorname{Pred}(A), \operatorname{ImPred}(B) \neq \operatorname{ImPred}(A)\}$

- the brothers : $\operatorname{brother}(A)=\{B \in \mathcal{X} \mid \operatorname{ImPred}(B) \in \operatorname{Pred}(A), B \notin$ $\operatorname{Pred}(A), \operatorname{ImPred}(B)=\operatorname{ImPred}(A)\}$ 


\section{Dendrograms}

\section{Definition}

$\mathcal{X}$ is a dendrogram if and only if the set $\operatorname{Pred}(A)$ of the predecessors of $A$, with the order relation induced by $\subset$ is a total order.

The maximal element of this family is a summit, which is the unique summit containing $A$. The smallest element is $\operatorname{ImPred}(A)$, the father of $A$, which is unique.

The arcs point from each element of the dendrogram to its immediate successor.

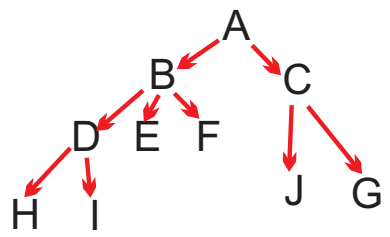

Figure: A dendrogram 


\section{Characterizations of a dendrogram}

The following properties are equivalent:

1) $\mathcal{X}$ is a dendrogram

2) $U, V, A \in \mathcal{X}: A \subset U$ and $A \subset V \Rightarrow U \subset V$ or $V \subset U$

3) $U, V \in \mathcal{X}: U \nsubseteq V$ and $V \nsubseteq U \Rightarrow U \cap V=\varnothing$ 


\section{A dendrogram of lakes}

Due to their particular properties, the closed balls of an ultrametric distance function form a dendrogram. Consider a particular closed ball $A=\operatorname{Ball}(p, \rho)$. We have to show that $\operatorname{Pred}(A)$ is completely ordered for

$C$. Consider two predecessors of $A$, a ball $B=\operatorname{Ball}(q, \lambda)$ and a ball $C=\operatorname{Ball}(s, \mu)$. As the node $p$ belongs to both balls $B$ and $C$, it is also center of these balls. Thus $B$ and $C$ are two balls with the same center $p$, and $\operatorname{Ball}(p, \lambda \wedge \mu) \subset \operatorname{Ball}(p, \lambda \vee \mu)$. 


\section{Creation of a dendrogram of lakes}




\section{The creation of lakes}

If the shortest path between $\Omega$ and $p$ passes through $q$ we have $\tau_{p}=\tau_{q} \vee d(p, q)$ if not $\tau_{p}<\tau_{q} \vee d(p, q)$.

\section{Lemma}

Any two nodes of an edge weighted graph verify $\tau_{p} \leq \tau_{q} \vee d(p, q)$.

Suppose that if $d(p, q)<\tau_{p}$. Then $d(p, q)<\tau_{p} \leq \tau_{q} \vee d(p, q)$ implies $\tau_{p} \leq \tau_{q}$. So $d(p, q)<\tau_{p} \leq \tau_{q}$ which similarly implies $\tau_{q} \leq \tau_{p}$. Hence $\tau_{p}=\tau_{q}$ which is compatible with the laws of hydrostatics.

\section{Lemma}

If two nodes $p$ and $q$ of an edge weighted graph verify $d(p, q)<\tau_{p}$ or $d(p, q)<\tau_{q}$, then $\tau_{p}=\tau_{q}$. 


\section{The creation of lakes}

\section{Lemma}

If an open ball Ball $(p, \lambda)$ has one node with a flooding level $\mu \geq \lambda$, then its flooding level is uniform and equal to $\mu$.

Proof: Suppose that the node $s$ in $\operatorname{Ball}(p, \lambda)$ verifies $\tau_{s} \geq \lambda$. The node $s$ as any node of an open ball is center of this ball. If $q \in \operatorname{Ball}(s, \lambda)$, we have $d(s, q)<\lambda \leq \tau_{s}$. Applying the preceding lemma yields $\tau_{s}=\tau_{q}$. As this is true for each node of $\operatorname{Ball}(p, \lambda)$, we have shown that the flooding level is constant and equal to $\tau_{s}$ on the entire ball $\operatorname{Ball}(p, \lambda)$. In particular if $\tau_{p}=\lambda$, then the flooding level in $\operatorname{Ball}(p, \lambda)$ is equal to $\lambda$. 


\section{The extension of the lakes}

Define $\varepsilon_{e}(X)$ : the lowest edge of the cocycle of $X$.

For $\tau_{p}=\lambda$, the flooding level on $\operatorname{Ball}(p, \lambda)$ is constant and equal to $\lambda$. As long as $\tau_{p}<\varepsilon_{e}(\operatorname{Ball}(p, \lambda))$, the extension of $\operatorname{Ball}\left(p, \tau_{p}\right)$ remains the same, the flooding is uniform and equal to $\tau_{p} ; \operatorname{Ball}\left(p, \tau_{p}\right)$ is a regional minimum, as all its nodes have the same weight, its the distance between its nodes is smaller than $\tau_{p}$, and its cocycle edges are higher than $\tau_{p}$.

For $\tau_{p}=\varepsilon_{e}(\operatorname{Ball}(p, \lambda))=\mu$ there exists an edge in the cocycle of $\operatorname{Ball}(p, \lambda)$ with a weight $\mu$. The closed ball $\operatorname{Ball}(p, \mu)$ strictly contains $\operatorname{Ball}(p, \lambda)$. As $\tau_{p}=\mu$, then for any other node $s$ in $\operatorname{Ball}(p, \mu)$ we have $\tau_{s} \leq \tau_{p} \vee d(p, s) \leq \mu$. However on $\operatorname{Ball}(p, \mu) \subset \operatorname{Ball}(p, \mu)$ the flood is constant and equal to $\tau_{p}=\mu$. 


\section{The extension of the lakes}

For any value $\sigma>\mu$, we have $\operatorname{Ball}(p, \mu) \subset \operatorname{Ball}(p, \sigma)$; if $\tau_{p}=\sigma$ the flood is constant on $\operatorname{Ball}(p, \sigma)$ and as $\operatorname{Ball}(p, \mu) \subseteq \operatorname{Ball}(p, \sigma)$ the flood also is constant on $\operatorname{Ball}(p, \mu)$.

In fact as long as $\sigma<\varepsilon_{e}(\operatorname{Ball}(p, \mu)), \operatorname{Ball}(p, \mu)=\stackrel{\operatorname{Ball}(p, \sigma)}{ }$.

\section{Lemma}

If there is at least one node with a weight $\mu$ in a closed ball $\operatorname{Ball}(p, \mu)$ of level $\mu$, all other nodes in this ball have a flooding level $\leq \mu$.

The diameter of $Y$ is $\lambda$. Such a closed ball is called lake zone of level $\lambda$, as the level of flooding inside is $\leq \lambda$. 


\section{The growing extension of a lake containing a particular}

node

The extension of the lakes containing a node $p$ as its flooding level $\eta$ increases is:

- for $\eta<\varepsilon_{\text {ne }} p$, the lake $X_{0}=\{p\}$ is a regional minimum lake.

- for $\eta=\varepsilon_{n e} p$, the lake $X_{1}=\operatorname{Ball}\left(p, \varepsilon_{n e} p\right)$ is a lake zone. The flood level is equal to $\eta$ on $X_{0}$ and $\leq \eta$ everywhere else on $X_{1}$. We have $\operatorname{diam}\left(X_{1}\right)=\varepsilon_{\text {ne }} p=\varepsilon_{e} X_{0}$.

- for $\operatorname{diam}\left(X_{1}\right)<\eta<\varepsilon_{e} X_{1}$, the lake $X_{2}=\operatorname{Ball}(p, \eta)$ is a regional minimum lake with the extension $X_{1}$.

- for $\eta=\varepsilon_{e} X_{1}$, the lake $X_{2}=\operatorname{Ball}\left(p, \varepsilon_{e} X_{1}\right)$ is a lake zone. The flood level is equal to $\eta$ on $X_{1}$ and $\leq \eta$ everywhere else on $X_{2}$. We have $\operatorname{diam}\left(X_{2}\right)=\varepsilon_{e} X_{1}$

- ...

- the alternating series of regional minima lakes and lake zones goes on until all nodes of $N$ are flooded. 


\section{Dominated flooding on a dendrogram}

We now define the level of the dominated flooding under $\omega$ on the dendrogram of the lakes. Define $\omega(X)$ the smallest value taken by the ceiling function $\omega$ on $X$. The lakes containing the node $p$ form an increasing series of nested sets $\kappa^{(n)}\{p\}$, the smallest being $\{p\}$, the largest being the root $\kappa^{(m)}\{p\}$ of the dendrogram.

The operator $\omega(X)$ is decreasing and the operator $\operatorname{diam}(X)$ increasing with $X$. As the series $\kappa^{(n)}\{p\}$ is increasing with $n$, we get a series of decreasing values $\omega\left(\mathcal{K}^{(n)}\{p\}\right)$ and a series of increasing values $\operatorname{diam}\left(\kappa^{(n)}\{p\}\right)$ :

a) as the set $\{p\}$ has no inside edge, we have $\operatorname{diam}\left(\kappa^{(0)}\{p\}\right)=\operatorname{diam}\{p\}=-\infty$. Hence $\omega\{p\}>\operatorname{diam}\{p\}=-\infty$

b) if $\kappa^{(m)}\{p\}$ is the root of the dendrogram and at the root we still have $\omega\left(\kappa^{(m)}\{p\}\right)>\operatorname{diam}\left(\kappa^{(m)}\{p\}\right)$, i.e. the ceiling of $p$ is higher than the root of the dendrogram, then $\tau_{p}=\omega\left(\kappa^{(m)}\{p\}\right)$ 

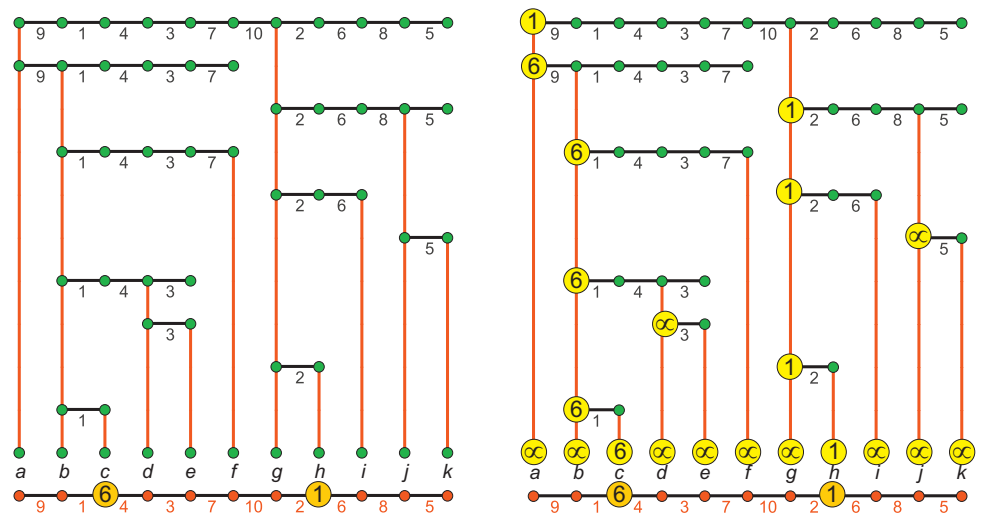

Figure: Left: Dendrogram associated to a MST. All nodes have a ceiling function equal to $\infty$ excepting the nodes $c$ and $h$, with values 6 and 1 .

Right: Each node of the dendrogram is assigned a ceiling value equal to the minimum ceiling value of all leaves it contains 


\section{Dominated flooding on a dendrogram}

c) In the other cases, there exists an index $k<m$ such that:

$\omega\left(\kappa^{(m)}\{p\}\right) \leq \operatorname{diam}\left(\kappa^{(m)}\{p\}\right)$, let $k \leq m$ be the smallest index for which $\omega\left(\kappa^{(k)}\{p\}\right) \leq \operatorname{diam}\left(\kappa^{(k)}\{p\}\right)($ rel. 1$)$

$\operatorname{diam}\left(\kappa^{(k-1)}\{p\}\right)<\omega\left(\kappa^{(k-1)}\{p\}\right) \leq \operatorname{diam}\left(\kappa^{(k)}\{p\}\right)=\varepsilon_{e}\left(\kappa^{(k-1)}\{p\}\right)$

The previous relation implies that $\tau_{\kappa^{(k-1)}\{p\}}=\omega\left(\kappa^{(k-1)}\{p\}\right.$ and on $\kappa^{(k)}\{p\}$ the maximal flooding level is $\operatorname{diam}\left(\kappa^{(k)}\{p\}\right.$.

In particular if $Y$ is a brother of $\kappa^{(k-1)}\{p\}$ then $Y$ is the root of a sub-dendrogram which may be processed independently, with $\omega(Y)=\operatorname{diam}\left(\kappa^{(k)}\{p\} \wedge \omega(Y)\right.$ 


\section{Dominated flooding on a dendrogram}

Each uncle $Y_{i}$ of $\kappa^{(k)}\{p\}$ with a father $\kappa^{(I)}\{p\}, I>k$ becomes the root of sub-dendrogram which may be processed independently, and with a ceiling level $\omega\left(Y_{i}\right)=\operatorname{diam}\left(\kappa^{(I)}\{p\} \wedge \omega\left(Y_{i}\right)\right.$.

This process cuts the upstream of $\kappa^{(k)}\{p\}$ in a number of sub-dendrograms which may then be processed independently one from another. 


\section{Illustration}
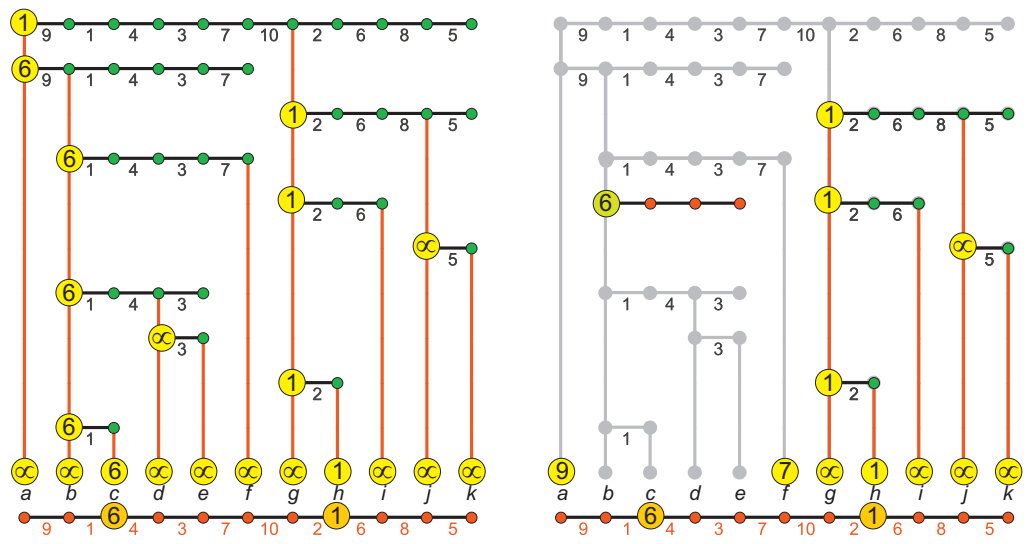

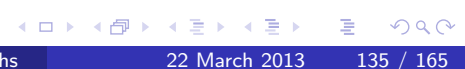


What about the lake containing the node $c$ ?The smallest index for which $\omega\left(\kappa^{(k)}\{c\}\right) \leq \operatorname{diam}\left(\kappa^{(k)}\{c\}\right)$, is $k=3$, with $\kappa^{(3)}\{c\}=[b, c, d, e, f]$ having a diameter 7 , whereas $\omega\left(\kappa^{(3)}\{c\}\right)=6$. For $k=2$, we get $\kappa^{(2)}\{c\}=[b, c, d, e]$ having a diameter 4, whereas $\omega\left(\kappa^{(2)}\{c\}\right)=6$. Hence: $\kappa^{(2)}\{c\}=[b, c, d, e]$ is $\tau_{c}=\tau_{\mathcal{K}^{(2)}\{c\}}=\omega\left(\kappa^{(2)}\{c\}\right)=6$ 

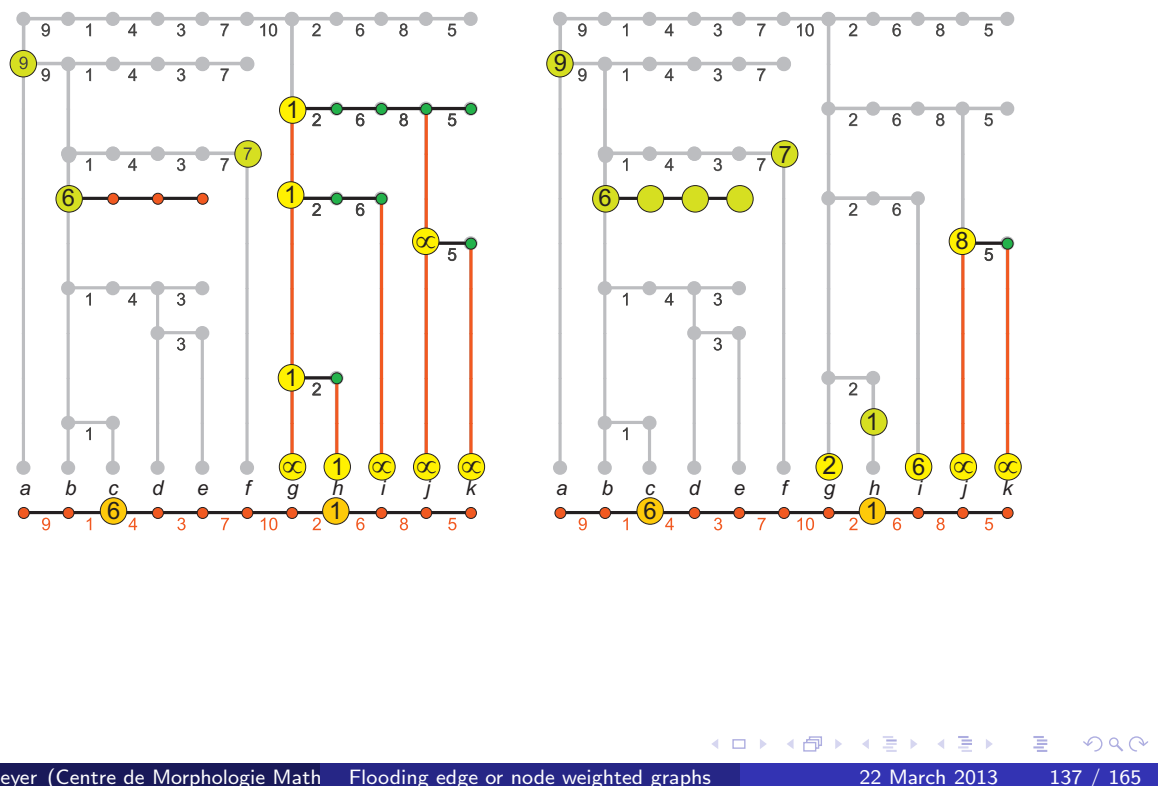
All ancestors of $\kappa^{(2)}\{c\}$ may be pruned. For $k>2, \kappa^{(k)}\{c\}$ is an ancestor of $c$, the flooding level of all its immediate successors which are not ancestors of $c$, that is, brothers of $\kappa^{(k-1)}\{c\}$ is lower or equal than $\operatorname{diam}\left(\kappa^{(k)}\{c\}\right)$. The edge linking each brother $Y$ of $\mathcal{K}^{(k-1)}\{c\}$ with its father $\kappa^{(k)}\{c\}$ is cut ; like that $Y$ becomes the root of a sub-dendrogram ; as its flooding level is lower or equal than $\operatorname{diam}\left(\kappa^{(k)}\{c\}\right)$, one sets $\omega(Y)=\omega(Y) \wedge \operatorname{diam}\left(\kappa^{(k)}\{c\}\right)$. On the same time all ancestors of $\kappa^{(2)}\{c\}$ and the edges linking them are suppressed. 
The set $\kappa^{(2)}\{c\}=[b, c, d, e]$ got its flooding level 6 and its upstream is pruned:

$-\kappa^{(3)}\{c\}=[b, c, d, e, f]$ is suppressed and the node $\{f\}$ becomes the root of sub-dendrogram, with a ceiling value $\omega(\{f\})=\omega(\{f\}) \wedge \operatorname{diam}\left(\kappa^{(3)}\{c\}\right)=7$. As the sub-dendrogram is reduced to a node, its ceiling value is its flooding value, 7 .

- $\kappa^{(4)}\{c\}=[a, b, c, d, e, f]$ is suppressed and the node $\{a\}$ becomes the root of sub-dendrogram, with a ceiling value $\omega(\{a\})=\omega(\{a\}) \wedge \operatorname{diam}\left(\kappa^{(4)}\{c\}\right)=9$. As the sub-dendrogram is reduced to a node, its ceiling value is its flooding value, 9 .

- $\kappa^{(5)}\{c\}=N$ is suppressed and the node $[g, h, i, j, k]$ becomes the root of sub-dendrogram, with a ceiling value $\omega([g, h, i, j, k])=\omega([g, h, i, j, k]) \wedge \operatorname{diam}\left(\kappa^{(5)}\{c\}\right)=1$. The ceiling value $\omega([g, h, i, j, k])$ of the root being known, flooding this subdendrogram becomes completely independent from the rest of the processing. 

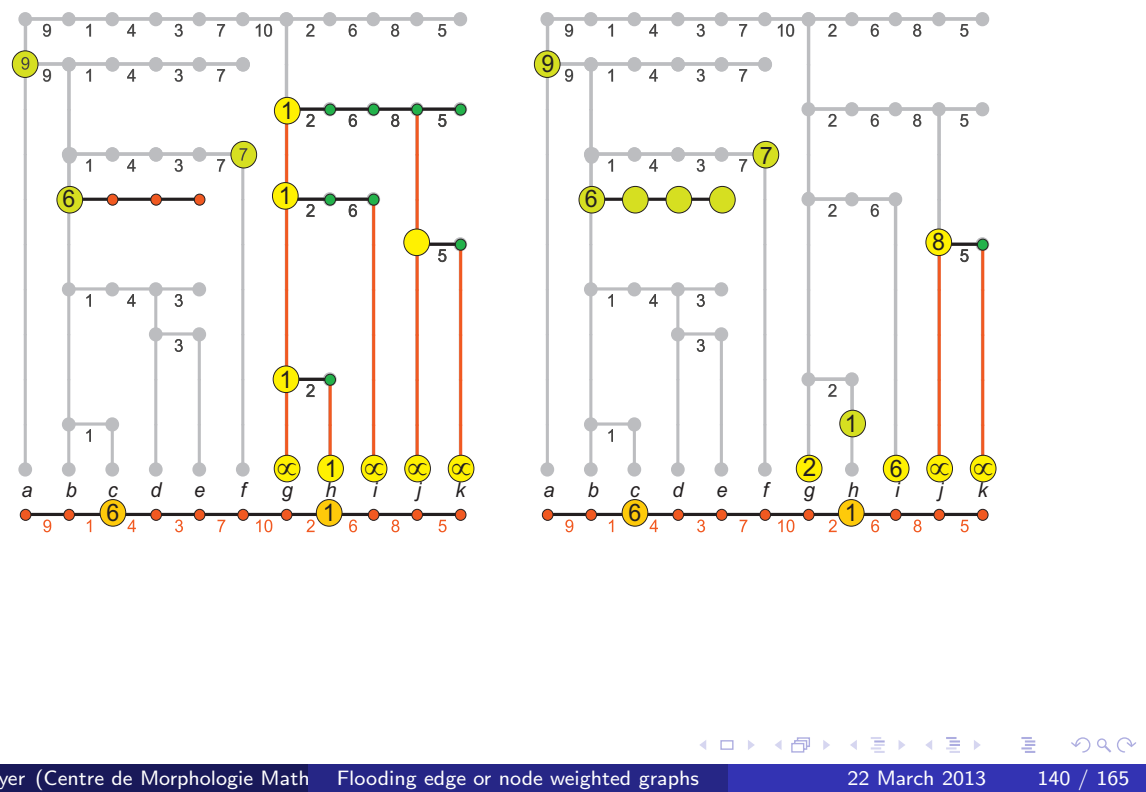
The smallest index for which $\omega\left(\kappa^{(k)}\{h\}\right) \leq \operatorname{diam}\left(\kappa^{(k)}\{h\}\right)$, is $k=1$, with $\kappa^{(1)}\{h\}=[g, h]$. The flooding level of $\kappa^{(0)}\{h\}=[h]$ is $\tau_{c}=\tau_{\mathcal{K}^{(0)}\{c\}}=\omega\left(\mathcal{K}^{(0)}\{h\}\right)=1$ and the upstream of $h$ can be pruned. - $\mathcal{K}^{(1)}\{h\}=[g, h]$ is suppressed and the node $\{g\}$ becomes the root of sub-dendrogram, with a ceiling value $\omega(\{g\})=\omega(\{g\}) \wedge \operatorname{diam}\left(\kappa^{(1)}\{h\}\right)=1$. As the sub-dendrogram is reduced to a node, its ceiling value is its flooding value: 1 .

- $\kappa^{(2)}\{h\}=[g, h, i]$ is suppressed and the node $\{i\}$ becomes the root of sub-dendrogram, with a ceiling value $\omega(\{i\})=\omega(\{i\}) \wedge \operatorname{diam}\left(\kappa^{(2)}\{h\}\right)=6$. As the sub-dendrogram is reduced to a node, its ceiling value is its flooding value, 6 . - $\mathcal{\kappa}^{(3)}\{h\}=[g, h, i, j, k]$ is suppressed and the node $[j, k]$ becomes the root of sub-dendrogram, with a ceiling value $\omega([j, k])=\omega([j, k]) \wedge \operatorname{diam}\left(\kappa^{(3)}\{h\}\right)=8$. The node $[j, k]$ being the root of a dendrogram with a ceiling value higher than its diameter gets flooded at the level of the ceiling value : 8 . This achieves the process since there are no more sub-dendrograms to process. 


\section{Contraction/expansion of flat zones and dendrogram flooding}




\section{Contracting inside edges of flat zones of node weighted graphs.}

$G_{n}$ : a node weighted graph with a ground level $f$ and a ceiling function $\omega$. Consider an edge $(p, q)$ such that $f_{p}=f_{q}$. Contracting this edge $=$ - suppressing the edge $(p, q)$

- merging both nodes into a new node $s$, with a ground value $f_{s}=f_{p}=f_{q}$ and a ceiling value $\omega_{s}=\omega_{p} \wedge \omega_{q}$

- suppress the edge linking a node $t$ with $p$ or $q$ and replace it with an edge $(t, s)$.

After contraction we get a new graph $G_{n}^{\prime}$ with a ground level $f^{\prime}$ and a ceiling function $\omega^{\prime}$ 


\section{Contracting inside edges of flat zones does not modify the dominated flooding}

$\tau$ : the highest flooding of $G_{n}$ under $\omega$

$\tau^{\prime}$ : the highest flooding of $G_{n}$ under $\omega^{\prime}$

We show that on all common nodes $\tau=\tau^{\prime}$ and that $\tau_{p}=\tau_{q}=\tau_{s}$

We first remark that replacing $\omega_{p}$ and $\omega_{q}$ by $\omega_{p} \wedge \omega_{q}$ does not change $\tau$. The shortest path linking $s$ in $G_{n}^{\prime}$ with $\Omega$ is the same as the shortest path linking $\Omega$ with $p$ or $q$ in $G_{n}^{\prime}$.

\section{Corollary}

All inside edges of flat zones may be contracted and produce each a unique node without changing the highest flooding of the graph. 


\section{Combining contractions and flooding on a dendrogram}

In the following figure A we want to construct the highest flooding of the red function under the green one. The contraction of the flat zones produces in fig. B a graph $G^{\prime}$ with 5 nodes $(a, b, c, d, e)$ with ground levels $(0,4,1,2,0)$ and ceiling levels $(0,5,3,3,1)$. The edges are then weighted with $\delta_{e n} n$, yielding the weights indicated in blue in fig.B. Fig.C presents the associated dendrogram. We first flood the node e. $\kappa^{(0)}(e)=\{e\}$. As $\operatorname{diam}\left(\mathcal{K}^{(0)}\{e\}\right)<\omega\left(\kappa^{(0)}\{e\}\right) \leq \operatorname{diam}\left(\mathcal{K}^{(1)}\{e\}\right)$, the flooding level of $e$ is $\omega\left(\kappa^{(0)}\{e\}=1\right.$.

$e$ has two brothers, the nodes $c$ and $d$, roots of subdendrograms reduced to 1 node. Having the same ceiling value, we have $\tau_{d}=\tau_{c}=\operatorname{diam}\left(\kappa^{(1)}\{e\} \wedge \omega(c)=2 \wedge 3=2\right.$.

$e$ has two uncles, the nodes $a$ and $b$, roots of subdendrograms reduced to 1 node. Their flooding value is $\tau_{a}=\operatorname{diam}\left(\kappa^{(1)}\{a\} \wedge \omega(a)=4 \wedge 0=0\right.$ and $\tau_{b}=\operatorname{diam}\left(\kappa^{(1)}\{b\} \wedge \omega(b)=4 \wedge 5=4\right.$ During the expansion, each node of the graph $G^{\prime}$ is replaced by the flat zone of the graph $G$ it represents, with identical flooding values, as illustrated in fig.F. 


\section{Illustration}
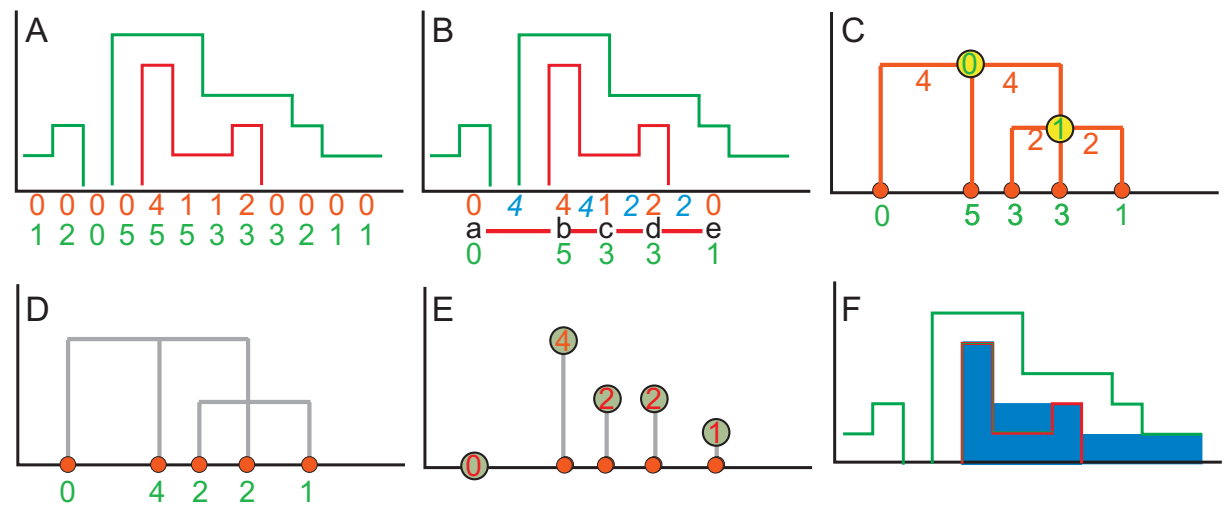

Figure: Creation of flat zones and flooding of the edge associated graph

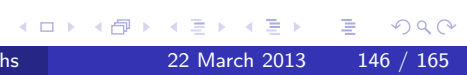




\section{Construction of the MST while contracting the edges in}

the flat zones.

Given a node weighted graph $G_{n}$ we assign to the edges weights equal to $\delta_{\text {en }} n$. It is beneficial to combine the construction of the MST of this and simultaneously contract the edges.

\section{Initialisation}

Create a tree with one node $p$ of the graph.

\section{Expansion}

As long as the tree does not contain all nodes of the graph:

Chose the lowest edge $(q, s)$ in the cocycle of $T$, such that $q \in T$ and $s \notin T$.

If $f_{q}=f_{s}$ : contract the edge $(q, s)$ on $q$ and link $q$ with the neighbors of $s$ not yet in $T$

Else : append the node $s$ to the tree: $T=T \cup\{s\}$ 
Relations between floodings on edge and node weighted graphs. 
Flooding a node weighted graph $=$ flooding an edge weighted graph

$G_{e}=$ edge weighted graph, $G^{n}=$ node weighted graph, $G_{e}^{n}=$ node and edge weighted graph For $G_{e}: \eta_{p}=\left(\varepsilon_{n e} e\right)_{p}$, i.e. the weight of the lowest edge adjacent to the node $p$.

\section{Theorem}

Consider $G_{n}$, a node weighted graph, and $G_{e}$, the derived edge weighted graph with edge weights $e=\delta_{\text {en }} n$. We then have the following equivalences: $\left\{\tau \geq n\right.$ e-flooding of $\left.G_{e}\right\} \Leftrightarrow\left\{\tau\right.$ n-flooding of $\left.G_{n}\right\}$ (eq- 1 )

This theorem has important algorithmic consequences. For constructing the highest flooding on the node weighted graph under a ceiling function $\omega$ we may construct the highest flooding of the edge weighted graph $G_{e}$ under $\omega$. 


\section{The waterfall flooding of an edge weighted graph}

Consider an edge weighted graph $G_{e}$. The waterfall flooding consists in assigning to each node of the graph a flooding level equal to the lowest adjacent edge: $\eta=\varepsilon_{n e} e$. The function $\eta$ is a particular flooding of $G_{e}$.

\section{Lemma}

In an edge weighted graph $G_{e}$, a function $\tau$ on the nodes is a valid flooding if and only if $\tau \vee \eta$ is a valid flooding: $\{\tau$ e-flooding of $\left.G_{e}\right\} \Leftrightarrow\left\{\tau \vee \eta\right.$ e-flooding of $\left.G_{n}\right\}$

This equivalence has the following consequence:

- by replacing $\tau_{p}<\eta_{p}$ by $\eta_{p}, \tau$ remains an e-flooding of $G_{e}$

- by replacing $\tau_{p}=\eta_{p}$ by $\tau_{p}^{\prime}<\eta_{p}, \tau^{\prime}$ remains an e-flooding of $G_{e}$ 


\section{Edge weighted graph invariant by $\gamma_{\mathrm{e}}$.}

Given $G_{e}$ : an edge weighted graph verifying $e=\gamma_{e} e=\delta_{e n} \varepsilon_{n e} e$. We create a node weighted graph $G^{\eta}$ with node weights $\eta=\varepsilon_{n e} e$.

As the edge weights verify $e=\delta_{e n} \varepsilon_{n e} e=\delta_{e n} \eta$ we have the equivalence 1 :

$\left\{\tau \vee \eta \geq \eta\right.$ e-flooding of $\left.G_{e}\right\} \Leftrightarrow\left\{\tau \vee \eta\right.$ n-flooding of $\left.G^{\eta}\right\}$.

Equivalence eq-2 : $\left\{\tau\right.$ e-flooding of $\left.G_{e}\right\} \Leftrightarrow\{\tau \vee \eta$ e-flooding of $\left.G_{e}\right\}$. Thus:

\section{Theorem}

Consider $G_{e}$, an edge weighted graph where each edge is the lowest edge of one of its extremities (invariant by $\gamma_{e}$ ), and $G^{\eta}$, the derived node weighted graph with node weights $\eta=\varepsilon_{n e} e$, we then have the following equivalences: $\left\{\tau\right.$ e-flooding of $\left.G_{e}\right\} \Leftrightarrow\left\{\tau \vee \eta\right.$ e-flooding of $\left.G_{e}\right\} \Leftrightarrow\{\tau \vee \eta$ n-flooding of $\left.G^{\eta}\right\}$. 


\section{Edge weighted graph invariant by $\gamma_{e}$.}

Consider now $\tau^{\prime} \geq \eta$ an e-flooding of $G_{e}$ which is also a n-flooding of $G_{\eta}$. Consider a subset of nodes $A$ of $N$. We define a new node distribution as follows:

- on $A: \tau \leq \eta$

- on $N / A: \tau=\tau^{\prime}$

This distribution verifies $\tau^{\prime}=\tau \vee \eta$. Hence, as stated in the preceding theorem, $\tau$ and $\tau^{\prime}$ are both e-floodings of $G_{e}$. 


\section{Case of a node weighted graph}

Given a node weighted graph $G^{n}$, we define $\varphi_{n} n=\varepsilon_{n e} \delta_{e n} n=\eta$. On the other hand we assign to the edges the weights $e=\delta_{e n} n=\delta_{e n} \varepsilon_{n e} \delta_{e n} n=\delta_{e n} \varphi_{n} n=\delta_{e n} \eta$.

The graph $G \eta$ is a flooding graph as $\eta=\varepsilon_{\text {ne }} e$ and $e=\delta_{\text {en }} \eta$. The preceding results apply to the graphs $G_{e}, G^{\eta}$ and $G^{e}$. $\left\{\tau\right.$ n-flooding of $\left.G^{n}\right\} \Leftrightarrow\left\{\tau \geq n\right.$ e-flooding of $\left.G_{e}\right\} \Leftrightarrow\{\tau \vee \eta$ e-flooding of $\left.G_{e}\right\} \Leftrightarrow\left\{\tau \vee \eta\right.$ n-flooding of $\left.G^{\eta}\right\}$. 


\section{Case of dominated floodings}

\section{Theorem}

A flooding $\tau$ is the highest flooding of an edge weighted graph $G_{e}$ under a ceiling function $\omega$ if and only if $\tau \vee \eta$ is the largest flooding of $G_{e}$ under the ceiling function $\omega \vee \eta$.

\section{Theorem}

If $\chi$ is the largest flooding of $G_{e}$ under the ceiling function $\omega \vee \eta$, then $\chi \wedge \omega$ is the largest flooding of $G_{e}$ under the ceiling function $\omega$. 


\section{Combining contraction.expansion of flat zones and closing of the isolated regional minima}




\section{Case of a node weighted graph}

$G^{n}$ : a node weighted graph with ground level $f$ and ceiling level $\omega$. Contracting its flat zones produces a graph $G^{\prime}=K G$ with ground level $f$ and ceiling level $\omega_{1}$. Each regional minimum of $f$ becomes an isolated regional minimum.

The closing $\varphi_{n}$ in the graph $G^{\prime}$ fills each regional minimum to the level of its lowest neighbor.

$\left\{\tau\right.$ n-flooding of $G^{\prime n}$ dominated by $\left.\omega_{1}\right\} \Leftrightarrow\left\{\tau_{1}=\tau \vee \eta\right.$ n-flooding of $G^{\prime \eta}$ dominated by $\left.\omega_{1} \vee \eta\right\}$.

If we expand the nodes of $G^{\prime}$, replacing each node by the flat zone it represents, with the flood distribution $\tau_{1} \wedge \omega$, we get the flooding of $G$ dominated by $\omega$. 


\section{Contraction and closing of the isolated regional minima}

A commutative diagram illustrates the process.

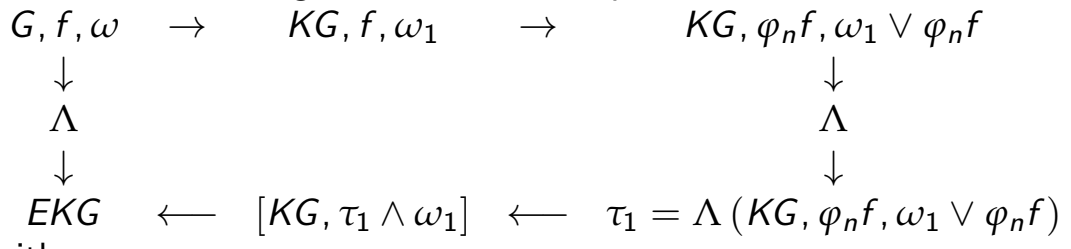

with 


\section{Contraction and closing of the isolated regional minima :}

\section{illustration}

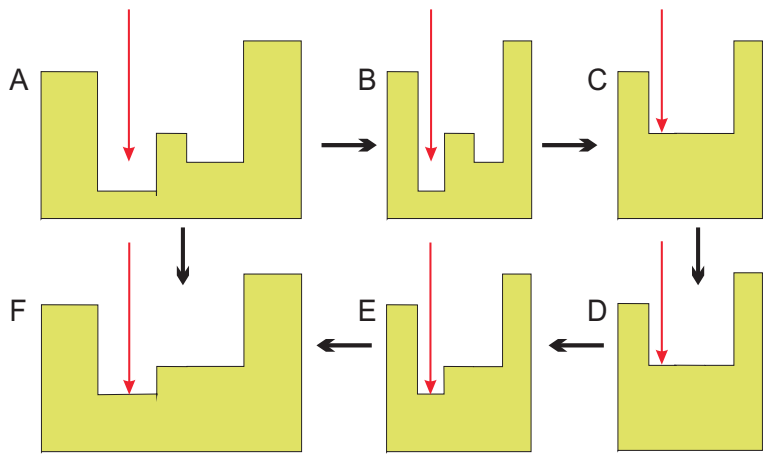




\section{Contraction and closing of the isolated regional minima :}

illustration

Fig.A: presents a topographic surface $f$ and a ceiling function equal to $\infty$ everywhere except at the position of the red arrow.

Fig. B: is obtained by contracting the flat zones with the associated ceiling function $\omega_{1}$, giving a graph $K G$

Fig. C: is obtained by the closing $\varphi_{n}$, which closes the isolated regional minima with a new ceiling function $\varphi_{n} f \vee \omega$

Fig.D: is the flooding $\tau$ of $\varphi_{n} f$ under $\varphi_{n} f \vee \omega$

Fig. E: the minimum $\tau_{1} \wedge \omega_{1}$ (which is also the flooding of the function in fig. B under the ceiling $\omega_{1}$ )

Fig.F: the expansion of fig. E yields the flooding of $f$ under $\omega$. 


\section{Cascading the preceding sequence}

The preceding sequence of transformations constructs a dominated flooding of a complex function thanks to the flooding of a simpler function. The same sequence may be applied for flooding this simpler function. And so on, producing a sequence of simpler and simpler functions to flood. 
Constructing a local flooding on a topographic graph 


\section{Fast and local flooding of a topographic surface.}

Using all results established above we propose an algorithm for local floodings.

$G_{n}$ a topographic graph, $f$ the ground level. We want to know the flooding at a given node $p$.for the dominated flooding of $f$ under $\omega$. Assigning to the edges the weights $\delta_{\text {en }} f$ produces a flooding graph: the lowest adjacent edge of each node has the same level than this node. If $p$ belongs to an isolated regional minimum and $\omega_{p} \leq\left(\varphi_{n} f\right)_{p}$, then $\tau_{p}=\omega_{p}$ If not, we flood the function $\varphi_{n} f$ under the ceiling function $\omega \vee \varphi_{n} f$ and get a flooding $\tau^{\prime}$. The desired flooding is $\tau=\tau^{\prime} \wedge \omega$. 


\section{Constructing the lake containing $p$}

For $X=\operatorname{Ball}\left(p, f_{p}\right)$ : if $\omega(X) \leq f_{p}$, then $p$ is in the upstream of a lake and is dry: $\tau_{p}=f_{p}$

If $\omega(X)>f_{p}$, we search the lake containing $p$ :

Until $\operatorname{diam}(X)<\omega(X) \leq \varepsilon_{e}(X)$ do $X=\kappa(X)=\operatorname{Ball}\left(p, f_{p}\right)$

The lake containing $p$ is $X$ at a level $\omega(X)$. 


\section{Procedure "up_hill"}

If $X$ is lake of the flooding, its uphill up to a level $\mu$ is constructed with the procedure "up_hill $(X, \mu)$ "

While $\varepsilon_{e}(X) \leq \mu$

$$
\begin{aligned}
& \lambda=\varepsilon_{e}(X) \\
& Y=\operatorname{Ball}\left(X, \varepsilon_{e}(X)\right)
\end{aligned}
$$

For each connected component $Z_{i}$ of $Y / X$ for which $\lambda>f_{Z_{i}}$ :

$$
\begin{aligned}
& \text { if } \omega\left(Z_{i}\right) \geq \lambda: \tau_{Z_{i}}=\lambda \\
& \text { else if } \left.\omega \text { is minimal at } q \text { in } Z_{i}: \text { apply "up-hill(Ball }\left(q, \omega_{q}\right), \lambda\right) \text { " }
\end{aligned}
$$

For each $p \in Y / X$ verifying $f_{p}=\lambda: \tau_{p}=\lambda$

$$
X=Y
$$

End While 


\section{Conclusion}

The comparison between floodings on edge weighted graphs and on node weighted graphs has given a better insight in both of them.

New algorithms have been derived allowing to chose the one is best suited for each application (type or processor, hardware, parallel processing, etc.) 